\title{
A novel Approach to Human Gait Recognition using possible Speed Invariant features
}

\author{
Anup Nandy* \\ Robotics and Artificial Intelligence Laboratory \\ Indian Institute of Information Technology Allahabad \\ Allahabad, Uttar Pradesh, 211012, India ${ }^{\dagger}$ \\ E-mail: nandy.anup@gmail.com \\ Rupak Chakraborty, Pavan Chakraborty, G.C. Nandi \\ Robotics and Artificial Intelligence Laboratory \\ Indian Institute of Information Technology Allahabad \\ Allahabad, Uttar Pradesh, 211012, India \\ E-mail: rupak97.4@gmail.com,\{pavan,gcnandi\}@iiita.ac.in \\ www.iita.ac.in
}

Received 24 September 2013

Accepted 18 May 2014

\begin{abstract}
In this paper a new area based technique is proposed for deriving gait signatures by decomposing the human body into three independent structural segments such as head node, arm swing and leg swing areas. Initially, all the feature points are represented as the sides of an n-sided polygon for calculating the area of each region. This technique induces surplus noise in the feature points which is in turn reflected in the human identification problem. This drawback inspires us to compute the area of each region by constructing a convex hull of the feature points in order to obtain certain key speed invariant features. Classification results demonstrate the ability of proposed feature extraction techniques using Bayes' classifier, distance metrics, and the proposed polynomial based distance metric. The performance analysis of various classifiers has been evaluated using Receiver Operating Characteristics (ROC) curve and the Cumulative Match Characteristics Curve (CMC) after performing N-fold cross validation technique.
\end{abstract}

Keywords: Human Gait; Polygon area; Convex Hull; Bayes’ Decision rule; Mahalanobis Distance; Polynomial based distance metrics.

\footnotetext{
* Typeset names in 10 pt Times Roman, uppercase. Use the footnote to indicate the present or permanent address of the author.

${ }^{\dagger}$ State completely without abbreviations, the affiliation and mailing address, including country typeset in $10 \mathrm{pt}$ Times italic.
} 


\section{Introduction}

In the modern world of computer technology, biometric recognition has proved its mettle in video surveillance applications which has attracted many researchers to invest their time in improving the security issues in order to make life safe and simple. The major challenges of current biometric systems include the automatic recognition of people with sensible error rates and producing results within a negotiable system speed and accuracy. Therefore, it is required to build a biometric system so robust that biometrics data collected from CCTV cameras can be analyzed automatically for making decisions in automated person recognition problems. Human gait biometric has tremendous potential to be considered as a powerful biometric tool for video surveillance systems [1-3]. In biomedical research human gait biometric could prove its ability in detection of gait related disorders in patients. Therefore, it is required to understand the normal human gait pattern. The Fig. 1 demonstrates a complete gait cycle for the normal walking pattern. The musculo-skeletal structure of human body [4-5] produces a human gait which requires extreme balance and stability. Every gait signal is repetitive in nature which supports the period of oscillation in stance and swing phase depicted in Fig.1. The complete gait cycle is accounted for the duration of successive heel strike of the same leg. In medical gait research, Murray et al. generated pathological gait patterns for normal persons [6] in order to detect abnormal gait patterns using those normal patterns. In Psychological studies, the Moving Light Display (MLD) was used [7] during the experiments to inquire about the gait perception using the human vision. Various studies of human gait research motivate the computer scientists to develop an automatic computer vision based gait biometric system.

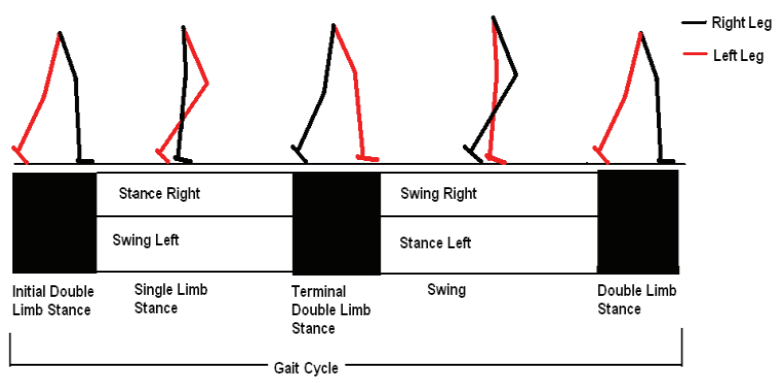

Fig. 1. Human gait period within a complete gait cycle [8]
The contribution of our research work is primarily based on investigating some key speed invariant features in the spatial domain for the purpose of human gait recognition. The simple feature selection techniques can be more effective in person identification problems using $\mathrm{n}$ - sided polygon and corresponding convex hull to calculate the area of head, arm and leg motion in the sagittal plane. Analyzing the three distinct regions of the human body in a hierarchical manner could produce statistically independent and identically distributed features. The classification technique emphasizes on the Naïve Bayes' rule with Multivariate Gaussian Distribution and other distance based metrics such as Mahalanobis distance, Euclidean distance etc. A novel classification approach using polynomial based distance metric has been proposed for the human recognition problem. The classifier performance has been evaluated statistically and results have been illustrated pictorially using Receiver Operating Characteristics Curve (ROC) and Cumulative Match Characteristics Curve (CMC) after applying $\mathrm{N}$-fold cross validation technique.

The main advantage of our gait recognition method is that it provides a separate analysis of three independent body segments with reference to the body shape centroid to the left and right of the sagittal plane. This analysis provides a clear insight into extracting speed invariant gait signatures. The investigation of each segment elucidates the temporal changes of human gait sequence during different gait speeds. The area based feature metrics captures temporal changes in feature points for different speeds.

The local, homogenous and cohesive region of interest for each independent body segment has been chosen to compute the area feature using convex hull and n-sided polygon based methods. This technique is very simple and intuitive to facilitate the strengths of recognition using locally embedded features. These features are characterized by local bounding points of each region which are used to analyze each segment separately. The advantage of using convex hull method is to find the geometrical area features of each region with minimum expensive operation. This method is fast and simple in computational practice. The area is calculated by comparison of feature pin points of each geometrical shape region (head node, arm swing and leg oscillation) and neglects those points which are far from the shape boundary. 


\section{Analysis of Previous Research}

In order to acquire information about the current research work on vision based human gait analysis, the following literature review has been provided with insights into the various model based and model free approaches for human identification problems.

\subsection{Model based approach}

The model based approach provides a way to derive static structural parameters of the human body. The potential advantage of model based approach is robustness in handling self-occlusion and insensitiveness to noise and change of apparels. The drawback of this approach is that high resolution images are required for identification which involves high computational complexity and gradually degrades the performance of gait recognition in outdoor environment. The literature review starts with a very simple method proposed by Lee and Grimson [9] for fitting ellipsoidal models to each segment of the human body structure. The model parameters were estimated for each region of the human silhouette. The combination of static and dynamic body features was identified by Wang et al. [10] in order to enhance classification results. Abdelkader et al. [11] suggested the static structural parameters of human body such as stride length and cadence for classification. Bobick and Johnson [12] proposed a simple model consisting of three sticks which represents two limbs and torso meeting at center of the pelvis point and measured the structural static parameters . The same three stick model was proposed by Davis and Taylor [13] with different feature extraction techniques. They have used dynamic features such as time duration of double limb support and the ratio of stance phase to swing phase. Yam et al. [14] proposed a dynamical gait model using coupled pendulum where the gait signatures were extracted from the angular rotation of knee and thigh. Niyogi et al. [15] demonstrated a simple human model for the study of human gait analysis in a spatio-temporal (XYT) space where the silhouette contour of the subject has been extracted to fit a stick model. Another approach to gait recognition was done by Cunado et al. [16] who proposed a simple model for both leg oscillations using a single line connected to the hip.

\subsection{Model free approach}

The model free approach depends on the moving shape and motion of the subject's body. The advantage of this approach is that recognition can be performed at large distances with low resolution images in outdoor environment. The model free approach is based on the extraction of human silhouette from the static background. The silhouette based [17] approach can be applied to derive model free gait signatures using the Gait Energy Image (GEI) which is the average of all the silhouette frames for a complete gait cycle. A statistical based technique using the Hidden Markov Model (HMM) has been applied to model the temporal sequences of human gait [18]. Another technique of gait recognition was based on the Principal Component Analysis [19] which was applied on 1D distance signals extracted from the series of silhouette image frames. It reduces dimensionality of feature space using the Eigen Space Transformation technique. The silhouette shape of the subject's motion was analyzed by Little et al. [20] for individual recognition. Sudeep Sarkar et al. [21] developed baseline algorithm for Human Id challenge problems where human recognition was accomplished by measuring the temporal correlation among background subtracted silhouette frames. The automatic human gait recognition was proposed by HayfronAcquah et al. [22] where gait symmetry was analyzed by subject's symmetrical properties to produce gait signatures. Akira Tsuji et al. [23] proposed a speed invariant model for human gait recognition using the silhouette based transformation technique.

The objective of introducing this research is not only to solve speed invariant person identification problems, but also a comprehensive study of human gait features has been emphasized to extract possible speed invariant gait signatures. Most of the speed invariant gait identification is based on feature based technique $[24,25]$ which is generally unable to explain the correctness and strengths of the features using a statistical technique. This inefficiency in features investigation might produce a large misclassification error rates during recognition time. The examination of features is extremely important before using it for classification purposes in order to reduce irrelevant information present in the features. Different gait speed 

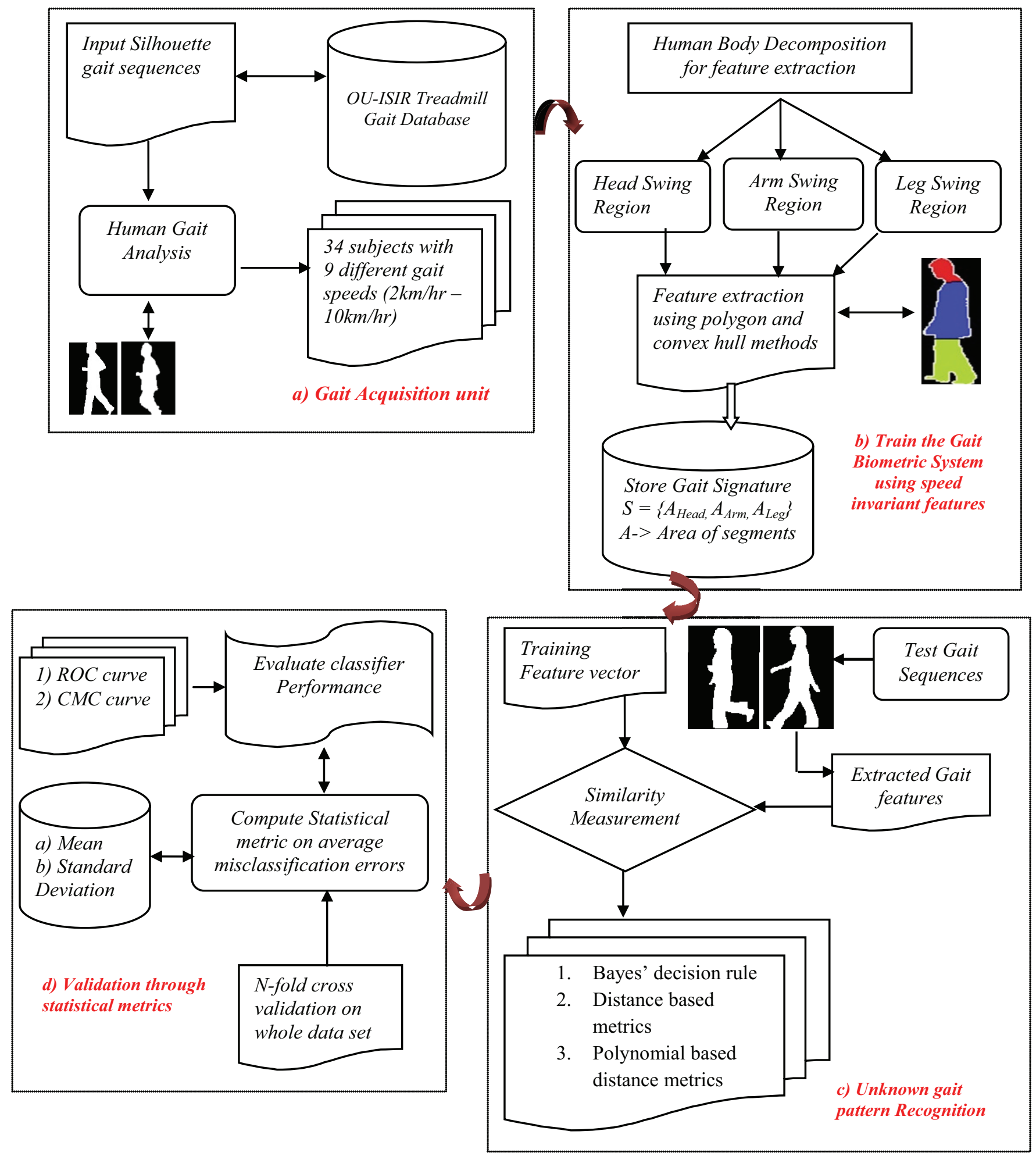

Fig.2. Process Model for Gait Identification System 
variations for each subject will attract a significant change in intra-class variance for separate gallery and probe gait sequence images. The analysis of discriminatory power of our feature vector for three independent segments of human body will provide an additional weightage to understand the maximum contribution of features for each region. A very simple and intuitive area based feature selection technique has been introduced for its mathematically tractability and efficiency in computational aspect with the convex hull method. The advantage of convex hull algorithm in area calculation demonstrates its time complexity for average and worst case, which are $\mathrm{O}(n \log n)$ and $\mathrm{O}\left(n^{2}\right)$ respectively irrespective of unsorted computed features. Moreover, it is practically accepted that though the error feature points lie outside the convex region it will not hamper much the connectivity of convex hull. It is a good practice to analyze each individual segment of human body to understand which body region carries most prominent gait signatures. This separate analysis will provide close insights into independently distributed features. The necessity of introducing this research work is to investigate whether the novel area based feature extraction technique could lead to correct classification and can convex hull and n-sided polygon based methods be used for providing speed invariant gait signatures? After a stringent analysis of gait features, this current research work will put forward the importance of convex hull method then n-sided polygon and the strengths of statistical based classifier in human identification with acceptable accuracy.

\section{Process Model for Gait Recognition System}

The process model, depicted in Fig. 2 demonstrates the sequential steps for the development of speed invariant human gait recognition system. This system architecture explains the brief overview of each separate block towards the original contribution of our research work.

\section{Feature Selection Process}

The most intriguing task in human gait recognition problem is to select intrinsic gait features. The human body is decomposed into three constituent structural segments in a hierarchical fashion in order to obtain identical and independently distributed features. We have obtained the Treadmill Gait database from OUISIR Gait lab [26] which is a collection of binary silhouette sequences for separate training and validation purposes. The feature selection process has been employed on this dataset (depicted in Fig.3) for 34 training subjects walking at 9 different gait speeds. The Features have been selected in the following manner.

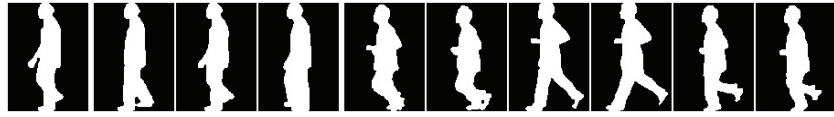

Fig.3. Sequence of silhouette frames at 9 different gait speeds

\subsection{Feature selection for the upper half}

- Arm swing area left of the sagittal plane.

- Arm swing area right of the sagittal plane.

- Head swing area left of the sagittal plane.

- Head swing area right of the sagittal plane.

\subsection{Feature selection for the lower half}

- Leg swing area to the left of sagittal plane.

- Leg swing area to the right of the sagittal plane.

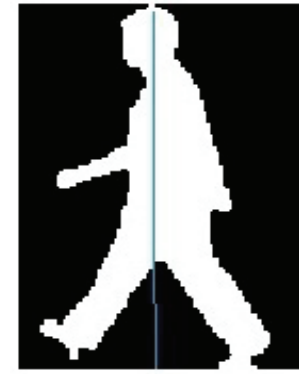

(a)

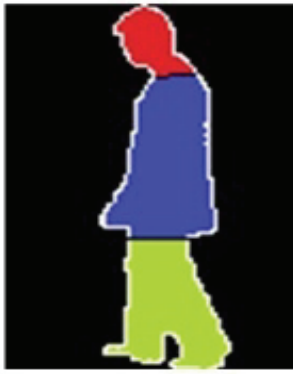

(b)
Fig. 4. (a) Silhouette image in sagittal plane

(b) Different regions of human body

The blue line in the Fig. 4.a shows the side view in the sagittal plane which bisects the human body in two planes. In Fig 4.b three distinct regions of human body have been represented by three different colors. This makes the feature selection independent of each other. The hierarchical process of feature selection has been depicted in Fig.5.

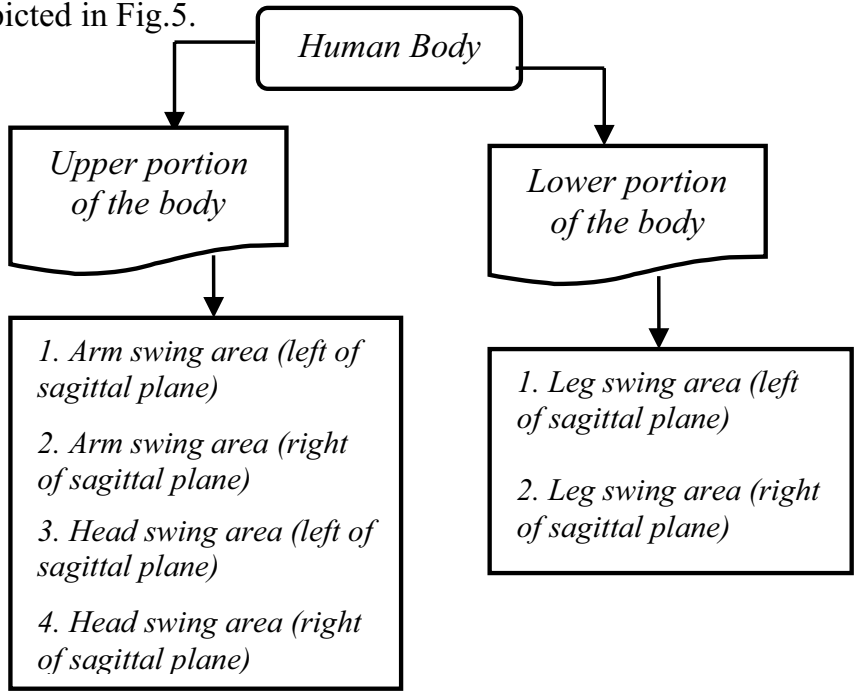

Fig. 5. Hierarchical process of feature selection 


\section{Feature Extraction Algorithm}

The feature extraction technique employs different algorithms to extract linear combination of features which provide us the maximum separability between the classes. The number of classes in this case implies the number of persons in training data set. We have applied two techniques to calculate the area of different segments of human body using polygon based approach and convex hull methods. The feature extraction algorithm with the calculation of area has been depicted with mathematical notations in the following manner. The area $A$ is calculated using shoelace formula.

$A=\frac{1}{2}\left|\sum_{i=1}^{n} x_{i}\left(y_{i+1}-y_{i-1}\right)\right|$

Where

$X=\left\{x_{1}, x_{2} \ldots x_{n}\right\}$

set of $x$ coordinates of the feature points

$Y=\left\{y_{1}, y_{2} \ldots y_{n}\right\}$

set of y coordinates of the feature points

Here $x_{i}$ and $y_{i}$ represent the $x$ and $y$ coordinates of the polygon vertices and convex hull respectively. This has been used as an algorithm for fast mathematical calculation. Area of the polygon has been calculated in the following way:

1. For each of the three different segments of the body depicted in (Fig 4.b) we take the coordinates of the pixel to extreme left of the sagittal plane where pixel value should be non zero. We collect $X=$ $\left\{x_{1}, x_{2}, \ldots x_{n}\right\}$ and $Y=\left\{y_{1}, y_{2}, \ldots y_{n}\right\}$ points for every frame of a given speed and repeat the same for all the nine different speeds.

2. It is required to remove the duplicate coordinates from the set $X$ and $Y$ to obtain non-repeating points in $X^{\prime}$ and $Y^{\prime}$. Using these coordinates we calculate the area of the polygon applying the shoelace formula.

3. The polygon is actually the locus of the point in each of the three different segments which have minimum $x$ coordinate value (in case of left swing area and $F(x, y) \neq 0$ ) and maximum $x$ coordinate value (in case of the right swing area $\&$ $F(x, y) \neq 0)$.

4. We are calculating the area of this polygon using the shoelace formula. In case of the convex hull the only difference is that we are taking the same set of points but we are calculating the convex set of those points. This is what is known as the convex hull and then we are calculating the area of the convex hull using the shoelace formula itself.

\section{Quick Hull algorithm for finding the convex hull}

The quick hull algorithm [27] is analogous to the quick sort algorithm which follows the general strategy of any divide and conquer algorithm.

1. Given a set of points $X=\left\{x_{1}, x_{2} \ldots x_{n}\right\}$ and $\mathrm{Y}=\left\{y_{1}, y_{2} \ldots y_{n}\right\}$ where $X$ and $Y$ represent the $x$ and $y$ coordinates of the set of points. Sort the points according to their increasing $x$ values where $x_{1}<x_{2}<x_{3}<x_{4} \ldots<x_{n}$.

2. Find the points with the maximum and minimum $x$ coordinate; these are the points which belong to the convex hull, let's call them $A$ and $B$.

3. Now join these two points with a straight line. This separates the whole set of points into two regions. One is to the left of the line segment joining $A \& B$ the other is to the right of the line joining $A \& B$.

4. Recursively call the quick hull algorithm for both these regions to the left and right of the line segment $A B$.

5. For each pass to the function find the point which has the maximum distance from $A B$. This point will also be included let's name it $C$ and join the ends of $A B$ to $C$ such that we get a triangle $A B C$. Now the points inside $A B C$ will not be inside the convex hull and hence are rejected.

6. Repeat the step 6 recursively for line segment $A C$ as well as $B C$.

7. Once we have all the points in the convex hull we calculate the area of that convex hull using the shoelace formula as already mentioned.
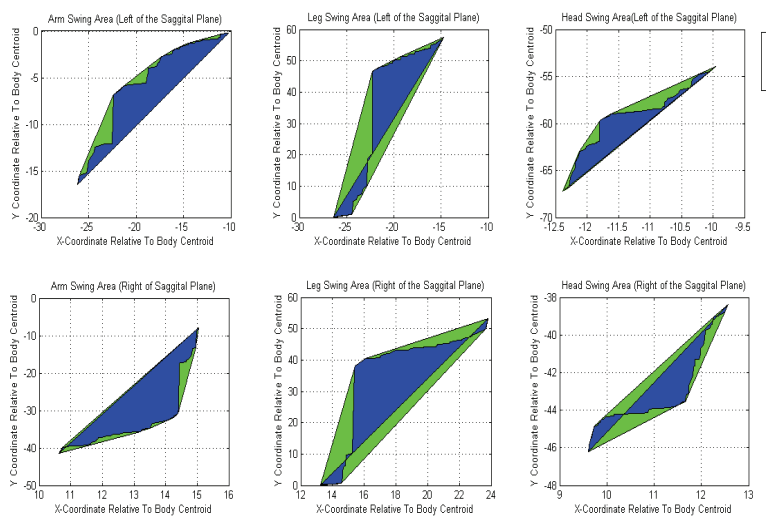

Fig.6. Illustration of feature points using polygon and convex hull shape for $2 \mathrm{~km} / \mathrm{hr}$. gait speed 

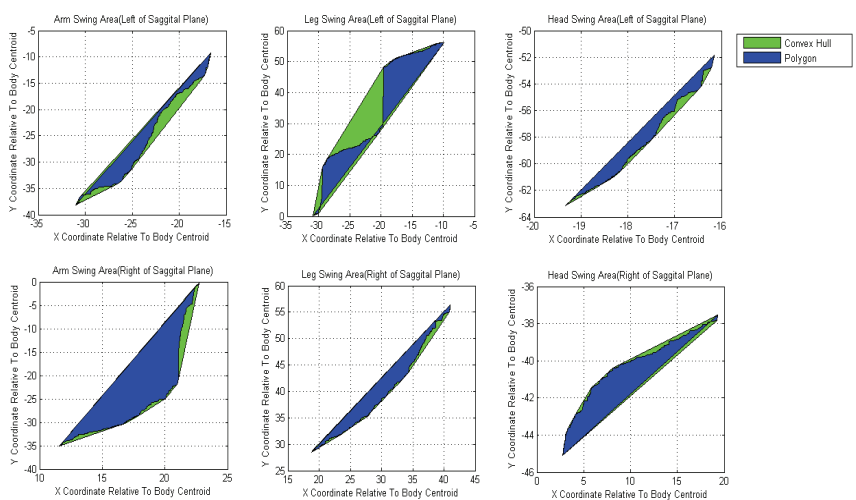

Fig.7. Illustration of feature points using polygon and convex hull shape for $10 \mathrm{~km} / \mathrm{hr}$. gait speed

The feature points for three different segments of human body (head, arm swing and leg swing) are shown in Fig. 6 and Fig. 7 for $2 \mathrm{~km} / \mathrm{hr}$ and $10 \mathrm{~km} / \mathrm{hr}$ gait speed. These graphs illustrate the polygon and convex hull shape made by those feature points. The blue and green color indicate the polygon and convex hull shape respectively for left and right of the sagittal plane of human body. It has been observed that the shape of the convex hull for upper body and lower body segments is being shortened while changing the gait speed. Henceforth the relevant feature points are held responsible to extract intrinsic body signatures from respective body segments.

\begin{tabular}{|c|c|}
\hline \multicolumn{2}{|r|}{ Mathematical Notations } \\
\hline$F(x, y)$ & Binary Image \\
\hline$\left(\sigma_{x}, \sigma_{y}\right)$ & Centroid \\
\hline$\sigma_{x}$ & $\frac{1}{n} \sum x_{i} ; \forall F(x, y) \neq 0$ \\
\hline$\sigma_{y}$ & $\frac{1}{n} \sum y_{i} ; \forall F(x, y) \neq 0$ \\
\hline$\phi(x, y)$ & set of points inside the body frames \\
\hline$\alpha(x, y)$ & $\begin{array}{l}\text { set of points in the portion of body } \\
\text { Fig. } 4(b) \text { by red, thus } \alpha(x, y) \subset \phi(x, y)\end{array}$ \\
\hline$\beta(x, y)$ & $\begin{array}{c}\text { set of points in the portion of body } \\
\text { Fig. } 4(b) \text { by blue, thus } \beta(x, y) \subset \phi(x, y)\end{array}$ \\
\hline$\delta(x, y)$ & $\begin{array}{c}\text { set of points in the portion of body } \\
\text { Fig. } 4(b) \text { by green, thus } \delta(x, y) \subset \phi(x, y)\end{array}$ \\
\hline$\pi(x, y)$ & $\begin{array}{l}\text { set of feature points in the portion of body } \\
\text { Fig. } 4(b) \text { by red, thus } \pi(x, y) \subset \alpha(x, y)\end{array}$ \\
\hline$\rho(x, y)$ & $\begin{array}{l}\text { set of feature points in the portion of body } \\
\text { Fig. } 4(b) \text { by blue, thus } \rho(x, y) \subset \beta(x, y)\end{array}$ \\
\hline$\tau(x, y)$ & $\begin{array}{l}\text { set of feature points in the portion of body } \\
\text { Fig. } 4(b) \text { by green, thus } \tau(x, y) \subset \delta(x, y)\end{array}$ \\
\hline$\hat{A}_{\pi}$ & Area of set of points in $\pi$ \\
\hline$\hat{A}_{\rho}$ & Area of set of points in $\rho$ \\
\hline$\hat{\hat{A}_{\tau}}$ & Area of set of points in $\tau$ \\
\hline
\end{tabular}

\section{Remarks:}

1. In all subsequent discussions, the left side of the sagittal plane will refer to the portion of the image to the left of the line $X=\sigma_{x}$

2. Similarly the right side of the sagittal plane will refer to the portion of the image to the right of the line $X=\sigma_{x}$

3. The area of the polygon that we have calculated is actually, the locus of the points which are at a maximum distance from the line $X=\sigma_{x}$ both to the left and right of the sagittal plane.

4. The convex hull is actually an optimization carried out on the set of feature points in order to augment the accuracy of our results.

5. Whenever we use the term area without explicitly specifying whether it is the area of the polygon or the convex hull, it is to be inferred that we are calculating both.

6. In result analysis, only the convex hull area has been used as an optimal metric of feature representation depicted in Fig. 20, 21, 22, 24. The polygon areas are not considered for redundant feature point selections.

\section{Algorithm 1: Feature Extraction technique}

Input: Raw input feature points of each segment.

Output: feature vector with six distinct features.

1: Begin

2: For each feature point

$\{X, Y\} \in[\alpha(x, y) \cup \beta(x, y) \cup \delta(x, y)] \approx S$

3: Do

$\pi_{L}(x, y), \rho_{L}(x, y)$

$=$ set of points at a maximum distance to the left of the sagittal plane and above the line $Y=\sigma_{y}$

$\pi_{R}(x, y), \rho_{R}(x, y)$

= set of points at a maximum distance to the right of the sagittal plane and above the line $Y=\sigma_{y}$ $\tau_{L}(x, y)=$ set of points at a maximum distance to the left of the sagittal plane and below the line $Y=\sigma_{y}$ $\tau_{L}(x, y)=$ set of points

to the left of the sagittal plane at a maximum distance and below the line $Y=\sigma_{y}$ 
4: End For

5: $\pi(x, y) \leftarrow \pi_{L}(x, y) \cup \pi_{R}(x, y)$

6: $\rho(x, y) \leftarrow \rho_{L}(x, y) \cup \rho_{R}(x, y)$

7: $\tau(x, y) \leftarrow \tau_{L}(x, y) \cup \tau_{R}(x, y)$

8: Do

9: Compute the area of polygon and convex hull separately formed by the set of points $\{X, Y\} \in S$ $\hat{A}_{\pi L} \leftarrow$ Area of set of points in $\pi_{L}$
$\hat{A}_{\pi R} \leftarrow$ Area of set of points in $\pi_{R}$
$\hat{A}_{\rho L} \leftarrow$ Area of the set of points in $\rho_{L}$
$\hat{A}_{\rho R} \leftarrow$ Area of the set of points in $\rho_{R}$
$\hat{A}_{\tau L} \leftarrow$ Area of the set of points in $\tau_{L}$ $\hat{A}_{\tau R} \leftarrow$ Area of the set of points in $\tau_{R}$

10: repeat for all the subjects at different gait speeds 11: The final feature vector $V=\left[\hat{A}_{\tau L}, \hat{A}_{\rho L}, \hat{A}_{\pi L}, \hat{A}_{\tau R}, \hat{A}_{\rho L}, \hat{A}_{\pi R}\right]^{T}$

The analysis of the feature space diagram provides an insight into the different movements of the human body segments with various gait speeds. The Fig. 8 illustrates the computed polygon area of head, arm and leg motions to the left and right of the sagittal plane where $X$-axis shows the speed and $Y$-axis shows the area of the polygon. The stability in the head swing area to the left of the sagittal plane has been observed at higher gait speeds. The abrupt changes in the leg and arm swing areas are also visualized which might be attributed to the physiological factors which influence the human gait cycle at higher gait speeds.
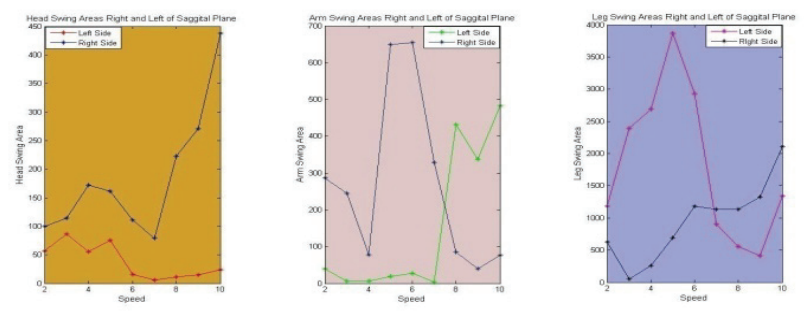

Fig. 8. Variation of features with speed for a single person using polygon area.
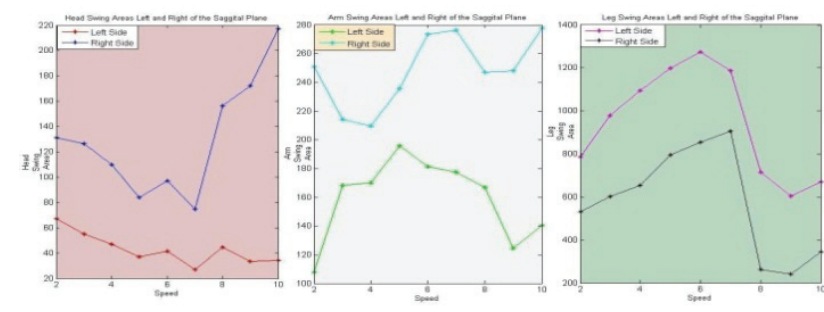

Fig. 9. Variation of features with speed for a single person using convex hull area.
The graph shown in Fig 9 illustrates the area of the convex hull formed by head, arm and leg swings to the left and right of the sagittal plane. $X$-axis shows the speed and the $Y$ axis shows the area of the convex hull formed by the locus of the points on the arm, leg, and head regions. It has been observed that the pair wise swing areas calculated over the head, arm and leg section are ideally anti-phase to each other.
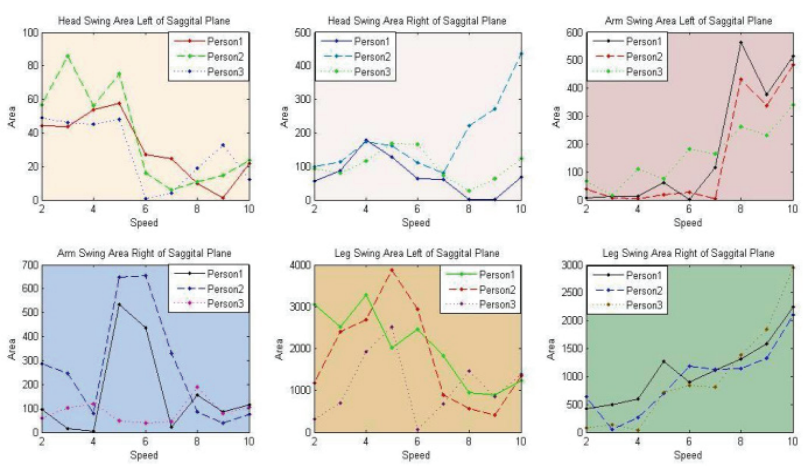

Fig. 10. Variation of features for three different persons at different speeds $2-10 \mathrm{~km} / \mathrm{hr}$. using polygon area
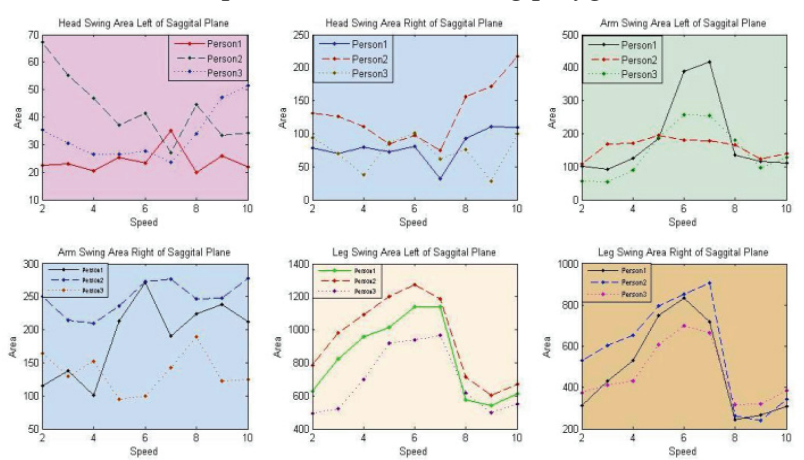

Fig. 11. Variation of features for three different persons at different speeds $2-10 \mathrm{~km} / \mathrm{hr}$. using convex hull area

The Fig.10 and Fig. 11 compare the distribution of the feature points for three different persons. The intrinsic speed invariant properties together with the uniqueness of feature points for different persons are illustrated in the feature space diagram. In Fig. 10, a higher degree of overlap in the feature space diagram has been observed as compared to Fig. 11. The wider inter-class separability provided by the convex hull area is also reflected in the experimental results. Hence, the juxtaposition of the feature points has enabled us to make a clear picture of the effects of different feature extraction techniques. The statistics of OU-ISIR gait dataset expresses 26 male and 8 female subjects with the age limit of 20's. The treadmill data set are probably healthy and they can walk and run on the treadmill. 


\section{Feature Analysis Process}

The individual discriminative power of each feature has been measured using Fisher's Discriminant Ratio (FDR) between two classes. This FDR value can be obtained irrespective of any distribution type for individual class. Let $\mu_{1}$ and $\mu_{2}$ be the means for two classes against an individual feature. Similarly $\sigma_{1}^{2}$ and $\sigma_{2}^{2}$ are the variances attached with each feature for two equiprobable classes. The FDR criterion is defined as

$$
F D R=\frac{\left(\mu_{1}-\mu_{2}\right)^{2}}{\sigma_{1}^{2}+\sigma_{2}^{2}}
$$

The feature with highest FDR value among the six computed feature's FDR value demonstrates the large difference between the inter class means and small variance in each intra class. The most informative feature is obtained through highest ranking feature through FDR criterion. There are also two conditions to determine the largest FDR value of two features using two metrics like absolute mean difference and sum of variance $\left(\sigma_{1}^{2}+\sigma_{2}^{2}\right)$. Features with similar absolute mean difference and lowest sum of variances produces largest FDR. On the other hand, the maximum absolute mean difference together with similar sum of variances of two features generates the highest FDR value. The FDR values against different subjects $S_{1}, S_{2}, \ldots S_{34}$ for extracted feature points of convex hull and polygon region have been depicted in Table 1 and 2 respectively.

Table 1. FDR value of feature points using convex hull

\begin{tabular}{ccccc}
\hline $\begin{array}{c}\text { Features } \\
\text { Name }\end{array}$ & $\begin{array}{c}\text { FDR } \\
\mathbf{S}_{\mathbf{1}}\end{array}$ & $\begin{array}{c}\text { FDR } \\
\mathbf{S}_{\mathbf{2}}\end{array}$ & $\begin{array}{c}\text { FDR } \\
\mathbf{S}_{\mathbf{3}}\end{array}$ & $\begin{array}{c}\text { FDR } \\
\mathbf{S}_{\mathbf{3 4}}\end{array}$ \\
\hline HL & 0.28 & 0.45 & 0.34 & 0.33 \\
HR & 0.08 & 0.13 & 0.16 & 0.16 \\
AL & 0.39 & 1.22 & 0.92 & 0.61 \\
AR & 0.44 & 0.79 & 0.67 & 0.75 \\
LL & 1.67 & 1.40 & 1.45 & 0.97 \\
LR & 2.19 & 1.28 & 2.42 & 1.58 \\
\hline
\end{tabular}

Table 2. FDR value of feature point using polygon area

\begin{tabular}{ccccc}
\hline $\begin{array}{c}\text { Features } \\
\text { Name }\end{array}$ & $\begin{array}{c}\text { FDR } \\
\mathbf{S}_{\mathbf{1}}\end{array}$ & $\begin{array}{c}\text { FDR } \\
\mathbf{S}_{\mathbf{2}}\end{array}$ & $\begin{array}{c}\text { FDR } \\
\mathbf{S}_{\mathbf{3}}\end{array}$ & $\begin{array}{c}\text { FDR } \\
\mathbf{S P}_{\mathbf{3 4}}\end{array}$ \\
\hline HL & 0.21 & 0.31 & 0.25 & 0.30 \\
HR & 0.12 & 0.11 & 0.15 & 0.14 \\
AL & 0.52 & 1.30 & 1.00 & 0.65 \\
AR & 0.65 & 0.66 & 0.66 & 0.56 \\
LL & 1.43 & 0.92 & 0.82 & 0.46 \\
LR & 2.14 & 1.28 & 1.81 & 1.05 \\
\hline
\end{tabular}

The largest FDR implies the more discriminatory power of the corresponding feature. It has been observed that the leg oscillations impart more discriminatory power than other parts of the body. The strength of the features has been measured using this technique where convex hull area produces largest FDR value for leg swing oscillations. The leg swing area can be considered as most leading features for both the cases i.e. polygon and convex hull areas. The less informative features are also included to empower the classification process because head node and arm swing movement will play an important role during changing in gait speed. The accuracy of the feature is quite impressive because the largest FDR value has provided the significance level of the each calculated features. The nomenclature of the feature names are: HL: Head Left, HR: Head Right, AL: Arm Left, AR; Arm Right, LL: Leg Left, LR: Leg Right Another statistical approach using hypothesis testing (ttest) has been used to measure the robustness of extracted features. The discriminatory power of each feature is evaluated by testing the significant difference between mean values of each feature for two classes. We have drawn normally distributed feature values with independent identically distributed (i.i.d) samples from each region of human body.

Let $x_{i}$ where $i=1,2,3, \ldots N$ be samples drawn from the class $C_{1}$ with mean value $m_{1}$

Let $y_{i}$ where $i=1,2,3, \ldots N$ be samples drawn from the class $C_{2}$ with mean value $m_{2}$

The similarity of mean values taken by a feature in two equiprobable classes has been decided by analyzing the following two tests for hypothesis.

$$
\begin{array}{ll}
H_{1}: & m_{1}-m_{2} \neq 0 \\
H_{0}: & m_{1}-m_{2}=0
\end{array}
$$

The first one is named as alternative hypothesis which indicates the significant changes in mean values of features for two classes. The second one is called as null hypothesis which defines a less significant change in

\begin{tabular}{|c|c|c|c|c|c|}
\hline \multicolumn{3}{|c|}{ Subject 1} & \multicolumn{2}{|c|}{ Subject 2} & \multirow{2}{*}{$\begin{array}{c}\text { Test } \\
\text { Statistic } \\
\left(T_{S}\right)\end{array}$} \\
\hline $\begin{array}{l}\text { Feature } \\
\text { Name }\end{array}$ & $\begin{array}{c}\text { Mean } \\
(\bar{x})\end{array}$ & $\begin{array}{c}\text { Variance } \\
\left(\sigma_{x}^{2}\right)\end{array}$ & $\begin{array}{c}\text { Mean } \\
(\bar{y})\end{array}$ & $\begin{array}{c}\text { Variance } \\
\left(\sigma_{y}^{2}\right)\end{array}$ & \\
\hline HL & 96.43 & 29.75 & 94.13 & 24.23 & 1.82 \\
\hline HR & 93.43 & 33.76 & 91.37 & 27.98 & 1.52 \\
\hline $\mathrm{AL}$ & 85.87 & 24.65 & 88.93 & 29.70 & 2.42 \\
\hline AR & 95.74 & 38.35 & 99.23 & 44.61 & 2.23 \\
\hline LL & 89.23 & 34.72 & 85.84 & 28.36 & 2.48 \\
\hline LR & 83.76 & 30.21 & 79.54 & 25.48 & 3.29 \\
\hline
\end{tabular}
mean values for two classes.

Table 3. Descriptive statistics of features for convex hull area 
Table 4. Descriptive statistics of features for polygon area

\begin{tabular}{cccccc}
\hline & Subject 1 & \multicolumn{2}{c}{ Subject 2 } & Test \\
\cline { 1 - 5 } Feature & Mean & Variance & Mean & Variance & Statistic \\
Name & $(\bar{x})$ & $\left(\sigma_{x}^{2}\right)$ & $(\bar{y})$ & $\left(\sigma_{y}^{2}\right)$ & $\left(\boldsymbol{T}_{\boldsymbol{S}}\right)$ \\
\hline HL & 101.42 & 35.12 & 99.10 & 33.11 & $1-63$ \\
HR & 105.51 & 46.23 & 102.77 & 37.49 & 1.74 \\
AL & 113.43 & 31.23 & 110.51 & 24.91 & 2.27 \\
AR & 117.36 & 56.54 & 113.89 & 51.21 & 1.94 \\
LL & 110.36 & 45.54 & 106.93 & 39.21 & 2.17 \\
LR & 111.32 & 53.87 & 107.21 & 47.65 & 2.37 \\
\hline
\end{tabular}

The more informative features are accepted if an alternative hypothesis deems to be true with a significance level $\sigma=0.05$ otherwise less informative features are taken with true test statistics of null hypothesis.

Let:

$\bar{x}$ and $\sigma_{x}^{2}$ are the mean and variance of class 1 .

$\bar{y}$ and $\sigma_{y}^{2}$ are the mean and variance of class 2 .

The test statistic is to be calculated in the following manner for unknown variance case:

$T_{s}=\frac{(\bar{x}-\bar{y})-\left(m_{1}-m_{2}\right)}{s_{z} \sqrt{\frac{2}{N}}}$

Where $s_{z}^{2}=\frac{1}{2}\left(\sigma_{x}^{2}+\sigma_{y}^{2}\right)$

The test statistics value has been checked with standard t-distribution table [28] with the hypothesis whether it is outside the closed interval or not. It has been seen that test statistic value of lower leg feature lies outside the closed interval of $[-2.032,2.032]$. We accept the $\mathrm{H}_{1}$ hypothesis where the significant mean difference occurs at the confidence level of 0.05 . The descriptive statistics value for convex hull and polygon based area are demonstrated in Table 4 and 5. The degree of freedom is closely related to number of subjects i.e. $N=34$

A pair-wise statistical test comparison has been done in $\left(\begin{array}{c}34 \\ 2\end{array}\right)$ combination and observed that the leg feature are more informative ones. The analysis of the feature makes a clear insight into the strengths and accuracy level of each individual feature.

The accuracy and the robustness of the extracted features has been statistically analyzed using hypothesis testing (t-test) as well as Fisher's Discriminant Ratio (FDR), the results given in tables 3,4 and tables 1,2 show the robustness and discriminative power of each feature independently. The features are robust in the sense that they can effectively capture the variations in the dynamic spatio-temporal gait signal. Since, we have used a bag of features approach; we must preclude the idea of testing each feature individually or a subset of features in order to identify its accuracy. The accuracy of the complete set of features has been well represented in the paper through various accuracy plots, ROC curves and CMC (Cumulative Match Characteristics) Curve.

The plots in figure 10 and figure 11, graphically illustrate how the selected set of features is able to accommodate the varying gait speeds of different persons. They depict the variation of the features for three different persons at nine different gait speeds from $2 \mathrm{~km} / \mathrm{hr}$. to $10 \mathrm{~km} / \mathrm{hr}$. Though the individual feature points for the three different persons are not linearly separable they show a wide variability which makes it practically as well as computationally tractable for identification purposes. The convex optimizations carried out on the set of feature points through the convex hull further augment the discriminative power of the features as illustrated through the various statistical tests.

The selected features quintessentially represent the inherent gait signatures of an individual. The combination of the swing areas both to the left and right of the sagittal plane are influenced by different latent physiological covariates like health, age, etc. This indeed leads to the identification of a unique person as we have seen through the FDR ratios in tables 1 and 2 which in essence measure the ratio of inter-class variation to intra-class variation.

An attempt has been made to project the gait signature in a 6-dimensional feature space and non-linear classifiers are used to create the decision boundaries between the different classes. Hence, the set of features are able to successfully identify a unique person with relatively low level of accuracy as discussed in the analysis of the classification algorithms.

\section{Classification Strategy}

The similarity measurement has been performed using extracted gait features obtained from sequence of silhouette frames. We have applied a multi-pronged strategy of classification using multivariate Gaussian distribution with Bayes' decision rule and distance based metrics such as Euclidean, City block, 


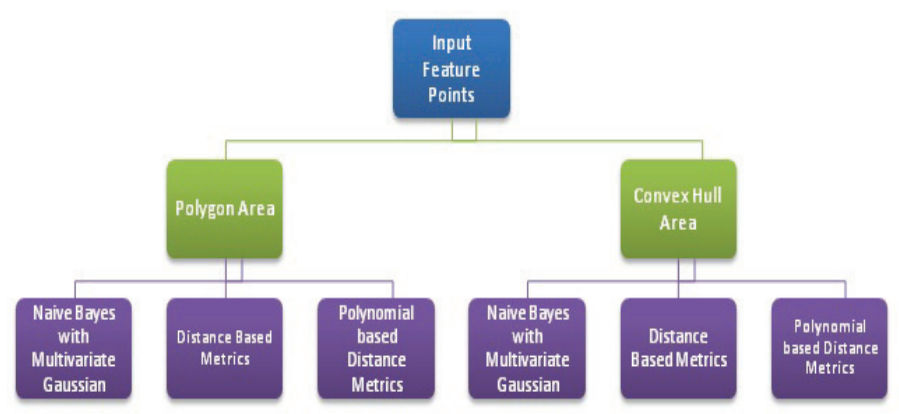

Fig.12. Description of Three Classification Techniques

Chebychev, Minkowski, and Mahalanobis distances. A novel approach for classification has been proposed using polynomial based distance metric generation. A unique polynomial is used to represent the features of the subject at different speeds, then the minimum distance between the input test vector and generated feature vector is found using various distance based metrics. It has been observed that the best fit for each feature of a given subject was satisfied by a polynomial of degree 5. Therefore, we have obtained 34 different polynomials for each different person. The pictorial representation of three classifiers is shown in Fig 12. The classification algorithms are described as follows.

\section{Algorithm 2: Bayes' Decision Rule for Classification}

Input: $C$ classes are given to be classified

Output: Closest class with less misclassification error

Step 1: Given $C$ number of Gait classes with prior probability. In our case $\mathrm{C}=34$

Step 2: Prior Probabilities $P_{1}, P_{2}, \ldots \ldots \ldots P_{C}$ for $C$ classes:

$$
\begin{aligned}
& 0 \leq P_{i} \leq 1 \\
& \sum_{i=1}^{C} P_{i}=1
\end{aligned}
$$

For every class the prior probability $P_{i}=\frac{1}{34}$;

Step 3: Define class conditional probability function

$$
\begin{aligned}
& f_{1}\left(\underline{x)}, f_{2}\left(\underline{x)}, \ldots \ldots \ldots \ldots \ldots f_{C}(\underline{x})\right.\right. \\
& \text { Where } \underline{x} \in \mathbb{R}^{N} \\
& \underline{x}=\text { input vector }
\end{aligned}
$$

$\left[x_{1}, x_{2}, \ldots \ldots \ldots, x_{N}\right]^{T} . N$ Dimensional feature vector For Naive Bayes' rule we use Multivariate Gaussian distribution which is given by:

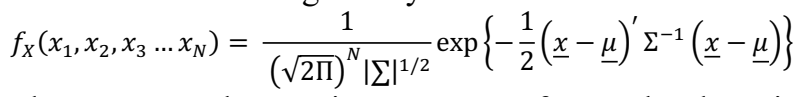

The mean and covariance vector for each class is already calculated from the training data.
Step 4: Calculate $f\left(x_{i}\right)$ using the input vector. We assign it to the class which has the maximum probability of containing that person using the following Naïve Bayes' decision rule.

$\operatorname{Max}\left[P_{1} \times f\left(x_{1}\right), P_{2} \times f\left(x_{2}\right), P_{3} \times f\left(x_{3}\right) \ldots P_{i} \times\right.$

$f(i) \forall i$; where $i=1: 34$ ]

Or

Put $\underline{x}$ in class $i$ if

$P_{i} f_{i}(\underline{x})>P_{j} f_{j}(\underline{x}) ; \forall j \neq i$. Where $\underline{x}=$ input vector extracted from test class

Step 5: Minimize the misclassification error probability.

Theorem 2. Let $\Omega$ be the set of all observations $\Omega \rightarrow$ Denotes the whole space

Let $\Omega_{1}, \Omega_{2}, \Omega_{3} \ldots \Omega_{C} \subseteq \Omega$ be such that

$\Omega_{i} \cap \Omega_{j}=\emptyset \quad \forall i \neq j$ and

$\mathrm{U}_{i=1}^{C} \Omega_{\mathrm{i}}=\Omega$

Let $D\left(\Omega_{1}, \Omega_{2}, \ldots \Omega_{C}\right)$ be a decision rule in $x \in \Omega_{i}$ Where $\Omega \subseteq \mathbb{R}^{\mathrm{m}}$ and $x \in \mathbb{R}^{m} \quad$ Set of all possible observations then $\underline{x} \rightarrow$ put in class $i$ If $D\left(\Omega_{1}, \Omega_{2}\right) \neq D\left(\Omega_{2}, \Omega_{1}\right)$;

$\Omega_{1}$ set $\rightarrow$ class 1 and $\Omega_{2}$ set $\rightarrow$ class 2

Probability of misclassification of $D\left(\Omega_{1}, \Omega_{2}, \ldots \Omega_{C}\right)$ denoted by

$\varepsilon\left(\Omega_{1}, \Omega_{2} \ldots \Omega_{C}\right)=\sum_{i=1}^{C} P_{i} \int_{\Omega_{i}{ }^{\prime}} f_{i}(\underline{x}) d \underline{x}$

The Bayes' decision rule minimizes the probability of misclassification $\varepsilon\left(\Omega_{1}, \Omega_{2} \ldots \Omega_{C}\right)$

over all the $D\left(\Omega_{1}, \Omega_{2}, \ldots \Omega_{C}\right)$.

\section{Algorithm 3: Distance based Classifiers}

Input: $C$ classes are given to be classified.

Output: Closest class with less misclassification error.

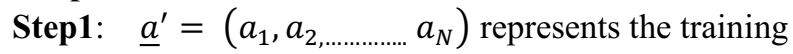
set in $\mathbb{R}^{N}$.

Step 2: Compute the mean of all the training data points in the training class $C_{i}$

Step 3: Repeat the steps for all the class $C_{i}$. Where $i=1: 34$

Step 4: The Euclidean/City Block/Chebychev/ Minkowski distance $d$ between centroid of each training classes and all the feature points in test class represented by $\underline{b}^{\prime}=\left(b_{1}, b_{2, \ldots} b_{N}\right)$ in $\mathbb{R}^{N}$ can be computed as:

$d_{p}(\underline{a}, \underline{b})=\left(\sum_{i=1}^{N}\left|a_{i}-b_{i}\right|^{p}\right)^{\frac{1}{p}} ; p \geq 1$

\section{Corollary:}


$p=2 \rightarrow$ Euclidean Distance (L2 Norm Distance)

$p=1 \rightarrow$ City Block Distance (L1 Norm Distance)

$p \rightarrow \infty$ Chebychev distance (Infinite Norm) using

$\lim _{p \rightarrow \infty} d_{p}(\underline{a}, \underline{b})=\underset{i=1,2,3}{\operatorname{argmax}}\left|a_{i}-b_{i}\right|$

$p>2 \rightarrow$ Minkowski Distance ( $p$ Norm Distance)

Step 5: Find the Mahalanobis distance between the training set $\underline{a}^{\prime}=\left(a_{1}, a_{2, \ldots \ldots \ldots \ldots . . . . . . .} a_{N}\right)$ and test set

$$
\underline{b}^{\prime}=\left(b_{1}, b_{2, \ldots} b_{N}\right) \text { in } \mathbb{R}^{N}
$$

Mahalanobis distance $\rightarrow \Delta^{2}=(\underline{a}-\underline{b})^{T} \Sigma^{-1}(\underline{a}-$

$\underline{b})$ Where $\Sigma$ is the covariance matrix of input dataset.

Step 6: Find the target class with minimum distance.

Theorem 3. Let $A=\mathbb{R}^{N} \rightarrow N$ dimensional Real Space Let $A \neq \emptyset$ where $A \rightarrow$ Non empty set and $\emptyset \rightarrow$

Empty set

Let $d: A \times A \rightarrow[0, \infty)$ where

$d$ denotes the distance function

$d(x, y) \rightarrow$ ordered pair

$d(x, y) \neq d(y, x)[$ may not be]

$d(x, y)$ takes value in the interval $[0, \infty)$.

Now $d$ is said to be a metric on $A$ if

1) $d(x, y)=d(y, x) \quad \forall x, y \in A$

2) $d(x, y)=0 \quad \leftrightarrow \quad x=y$

3) $d(x, y)+d(y, z) \geq d(x, z) \forall x, y, z \in A$

\section{Algorithm 4: Polynomial based distance metrics}

Input: $C$ classes are given to be classified.

Output: Closest class with less misclassification error.

Step 1: For each person we apply polynomial based distance metric using 6 polynomials. These 6 polynomials represent the six different features for each person. So in total we have $34 \times 6$ polynomials.

Step 2: The polynomials are fitted using the method of least squares in order to find the best fit and the values of the coefficients are found using the Vander Monde matrix, whose elements are the powers of the variable.

Step 3: A five degree polynomial is given by:

$f(x)=a_{0} x^{5}+a_{1} x^{4}+a_{2} x^{3}+a_{3} x^{2}+a_{4} x^{1}+a_{5}$

Where the coefficients represent the interdependence between the speed and the particular feature here $x$ denotes the speed of the person.

$$
\begin{aligned}
& f_{1}(x)=a_{0} x^{5}+a_{1} x^{4}+a_{2} x^{3}+a_{3} x^{2}+a_{4} x^{1}+a_{5} \\
& f_{2}(x)=b_{0} x^{5}+b_{1} x^{4}+b_{2} x^{3}+b_{3} x^{2}+b_{4} x^{1}+b_{5}
\end{aligned}
$$

$$
\begin{gathered}
f_{3}(x)=c_{0} x^{5}+c_{1} x^{4}+c_{2} x^{3}+c_{3} x^{2}+c_{4} x^{1}+c_{5} \\
f_{4}(x)=d_{0} x^{5}+d_{1} x^{4}+d_{2} x^{3}+d_{3} x^{2}+d_{4} x^{1}+d_{5} \\
f_{5}(x)=e_{0} x^{5}+e_{1} x^{4}+e_{2} x^{3}+e_{3} x^{2}+e_{4} x^{1}+e_{5} \\
f_{6}(x)=k_{0} x^{5}+k_{1} x^{4}+k_{2} x^{3}+k_{3} x^{2}+k_{4} x^{1}+k_{5}
\end{gathered}
$$

\section{Step 4:}

Now for given input vector $X=\left[x_{1}, x_{2}, x_{3}, x_{4}, x_{5}, x_{6}\right]^{T}$ we generate our own feature vector for that given speed (say $3 \mathrm{kmph}$ ) and repeat the same for all the classes using the polynomial given above:

$$
G=\left[\begin{array}{l}
f 1(3) \\
f 2(3) \\
f 3(3) \\
f 4(3) \\
f 5(3) \\
f 6(3)
\end{array}\right]
$$

Step 5: Now we use different distance based metrics to find the distance between these two vectors. The class for which we get the minimum distance is the class to which person is correctly classified.

The three algorithms serve as baseline algorithms for validating our feature set, they are used together to reflect the inherent heteroscedastic nature of the dataset and to carry out a comparative study of how different non-linear classifiers fare in the same problem domain. The use of different distance metrics in both the Nearest Neighbour and the Polynomial based classifiers elucidate how different distances impact the classification results. As an obvious and intuitive explanation distance metrics which have an elliptical region of influence (i.e. Mahalanobis distance) outperform those distance metrics which have a spherical region of influence (i.e. Euclidean), this is because of their inherent ability to accommodate the outliers in the dataset. The naïve Bayes' classifier is used to handle the nonlinearity problem in time varying gait signal using multivariate Gaussian distribution. It is being used for its easy tractability in mathematical modeling and it supports the central limit theorem on extracted i.i.d. gait features.

\section{Results Analysis and Discussion}

In order to perform human gait analysis, separate gait datasets for training and validating have been used in this research work. The classification result obtained from various classifiers provides us an insight into the 
performance of the speed invariant gait biometric system. The selection of optimal feature points produces more promising results using the convex hull technique. It overcomes the drawback of the polygon area which generates the concave area with those feature points and hence leads to incorporation of noise in the datasets. The following tables illustrate the various classification results obtained using convex hull and polygon area. Table5 depicts the classification accuracy for various distance metrics using the convex hull area. Table 6 represents the classification accuracy for various distance based metrics using polynomial fitting and convex hull area. Table 7 illustrates the classification accuracy for various distance based metrics using the polygon based area.

Table 5. Distance Metric using convex hull

\begin{tabular}{cc}
\hline Distance Metric & Accuracy (\%) \\
\hline Mahalanobis & 60 \\
City Block & 55 \\
Chebychev & 42 \\
Euclidean & 52 \\
\hline
\end{tabular}

Table 6. Polynomial based distance metrics using convex hull

\begin{tabular}{lc}
\hline \multicolumn{1}{c}{ Distance Metric } & Accuracy (\%) \\
\hline Mahalanobis & 56.29 \\
Euclidean & 54.07 \\
Minkowski (with power 5) & 51.85 \\
Minkowski (with power 6) & 51.53 \\
Minkowski (with power 7) & 51.11 \\
City Block & 52.59 \\
Chebychev & 50.23 \\
\hline
\end{tabular}

Table 7. Distance metrics using polygon area

\begin{tabular}{lc}
\hline \multicolumn{1}{c}{ Distance Metric } & Accuracy (\%) \\
\hline Mahalanobis & 36.34 \\
Euclidean & 31.04 \\
Minkowski (with power 5) & 28.43 \\
Minkowski (with power 6) & 27.45 \\
Minkowski (with power 7) & 27.40 \\
City Block & 34.96 \\
Chebychev & 27.78 \\
\hline
\end{tabular}

Similarly the classification results for various polynomial based distance metrics with polygon area has been depicted in Table 8. On the other hand, the classification result using multivariate Gaussian distribution with Bayes' decision rule for polygon based area and convex hull area has been illustrated in table 9 .

Table 8. Polynomial based distance metrics using Polygon area

\begin{tabular}{lc}
\hline Distance Metric & Accuracy (\%) \\
\hline Mahalanobis & 44.183 \\
City Block & 38.627 \\
Chebychev & 33.379 \\
Euclidean & 35.359 \\
\hline
\end{tabular}

Table 9. Bayes' rule classifier using polygon and convex hull area

\begin{tabular}{ccc}
\hline $\begin{array}{c}\text { Probabilistic } \\
\text { Classifier }\end{array}$ & $\begin{array}{c}\text { Accuracy (\%) } \\
\text { using polygon } \\
\text { area }\end{array}$ & $\begin{array}{c}\text { Accuracy (\%) } \\
\text { using convex hull } \\
\text { area }\end{array}$ \\
\hline $\begin{array}{c}\text { Bayes' decision } \\
\text { rule }\end{array}$ & 48.56 & 60.32 \\
\hline
\end{tabular}

The performance of various classifiers is of prime importance to make a robust gait biometric system in order to solve the person identification problem. The standard statistical metrics are used to evaluate the robustness of each classifier in the biometric field. We have applied $\mathrm{N}$-fold cross validation technique which uses Leave one out method to validate the classification results. The following algorithm 5 explains the working principal of $\mathrm{N}$ - fold cross validation technique. We have plotted the misclassification error rates for each classifier. The best classifier can be declared as a distinct clear cut winner if the mean is less and standard deviation is also less on misclassification error rates after obtaining cross-validation results.

The advantage of cross validation is to avoid the random portioning of original dataset and each subset partition is sequentially used for both training and validation which maintains the overall variability of the original data set without any loss of generality.

\section{Algorithm 5: N- fold cross validation technique}

Input: Entire population of feature vectors $V$

Output: Best classifier with less mean and less std.

Step 1: The original sample of 34 subjects is divided into $N$ subsamples with equal data size. Here $N=9$ for each gait speed changing from $2 \mathrm{~km} / \mathrm{hr}$ to $10 \mathrm{~km} / \mathrm{hr}$.

Step 2: The $N$ subsamples can be represented as $\left[A_{1}, A_{2}, \ldots A_{9}\right]$ where a single subsample used as a test data and remaining $N-1$ samples considered as training data.

Step 3: Apply Leave one out method for $N$ fold cross validation

$$
\begin{aligned}
A 1 \rightarrow & \text { validation set and } A_{2} \cup A_{3}, \ldots A_{9} \rightarrow \\
& \text { Training set. } \\
A 2 \rightarrow & \text { validation set and } A_{1} \cup A_{3}, \ldots A_{9} \rightarrow \\
& \text { Training set. } \\
A 9 \rightarrow & \text { validation set and } A_{1} \cup A_{2}, \ldots A_{8} \rightarrow \\
& \text { Training set. }
\end{aligned}
$$

Co-published by Atlantis Press and Taylor \& Francis Copyright: the authors 
Step 4: repeat the step 3 for $N$ times where each subsample is used as the validation set exactly once.

Step 5: Obtain 9 misclassification error rates for each classifier.
Step 6: Calculate the mean and standard deviation of 9 misclassification error rates.

Step 7: If mean is less and standard deviation is less then declares the classifier as the Best classifier.

Table 10. Polygon coefficient values obtained for a single subject using polygon area

\begin{tabular}{lllllll}
\hline \multicolumn{1}{c}{ Features } & \multicolumn{1}{c}{$\mathbf{A}_{\mathbf{0}}$} & \multicolumn{1}{c}{$\mathbf{A}_{\mathbf{1}}$} & \multicolumn{1}{c}{$\mathbf{A}_{\mathbf{2}}$} & \multicolumn{1}{c}{$\mathbf{A}_{\mathbf{3}}$} & \multicolumn{1}{c}{$\mathbf{A}_{\mathbf{4}}$} & \multicolumn{1}{c}{$\mathbf{A}_{\mathbf{5}}$} \\
\hline Leg Swing(Left) & -10.65 & 315.10 & -3468.96 & 17454.05 & -39571.37 & 34221.60 \\
Arm Swing(Left) & 0.17225 & -7.17 & 102.71 & -629.83 & 1687.68 & -1525.83 \\
Head Swing(Left) & 0.139 & -4.26 & 49.42 & -268.06 & 664.19 & -543.22 \\
Leg Swing(Right) & -3.02 & 96.27 & -1143.22 & 6248.79 & -15215.61 & 13670.41 \\
Arm Swing(Right) & -0.312 & 8.98 & -90.60 & 365.22 & -475.77 & 392.09 \\
Head Swing(Right) & -0.76 & 23.37 & -270.26 & 1442.59 & -3468.13 & 3082.91 \\
\hline
\end{tabular}

Table 11. Polygon coefficient values obtained for a single subject using convex hull area

\begin{tabular}{cllllll}
\hline Features & \multicolumn{1}{c}{$\mathbf{A}_{\mathbf{0}}$} & \multicolumn{1}{c}{$\mathbf{A}_{\mathbf{1}}$} & \multicolumn{1}{c}{$\mathbf{A}_{\mathbf{2}}$} & \multicolumn{1}{c}{$\mathbf{A}_{\mathbf{3}}$} & \multicolumn{1}{c}{$\mathbf{A}_{\mathbf{4}}$} & \multicolumn{1}{c}{$\mathbf{A}_{\mathbf{5}}$} \\
\hline Leg Swing(Left) & 0.197 & -1.879 & -27.588 & 378.191 & -1239.982 & 1975.128 \\
Arm Swing(Left) & 0.135 & -1.879 & -7.263 & 192.940 & -756.757 & 950.245 \\
Head Swing(Left) & -0.088 & 2.621 & -29.658 & 160.391 & -416.574 & 468.079 \\
Leg Swing(Right) & 0.552 & -12.381 & 90.102 & -239.266 & 245.220 & 439.050 \\
Arm Swing(Right) & 0.066 & -0.719 & -7.235 & 111.328 & -365.999 & 524.468 \\
Head Swing(Right) & -0.112 & 3.505 & -40.694 & 216.564 & -513.097 & 521.710 \\
\hline
\end{tabular}

The polynomial coefficient values of a single subject (depicted in table 10 and table 11) are provided for the better understanding of polynomial based distance metrics. The Fig.13 illustrates the misclassification error rates for the Naïve Bayes' classifier using the polygon area as well as the convex hull area. It is well understood from the graph that the convex hull area gives less misclassification error rate as compared to the polygon based area. This result has been obtained by the $\mathrm{N}$ - fold cross validation with leave one out metric, where $\mathrm{N}=9$ (in our case). The following table 12 shows the statistical metrics obtained for the Naïve Bayes' using both areas.

Table 12. Statistical measurement for Naïve Bayes' rule

\begin{tabular}{ccc}
\hline Measure of Area & Mean & Standard Deviation \\
\hline Polygon & 48.5294 & 6.67 \\
Convex Hull & 39.84125 & 10.3058 \\
\hline
\end{tabular}

The figure 14 shows the misclassification error rates for the different distance metric based classifiers. It is clearly understood from the graph that the Mahalanobis distance provides the minimum misclassification error rate. We attribute this fact to the distance metric's incredible ability to consider the outliers and its apparent similarity with the Multivariate Gaussian Distribution. The Mahalanobis distance has emerged as

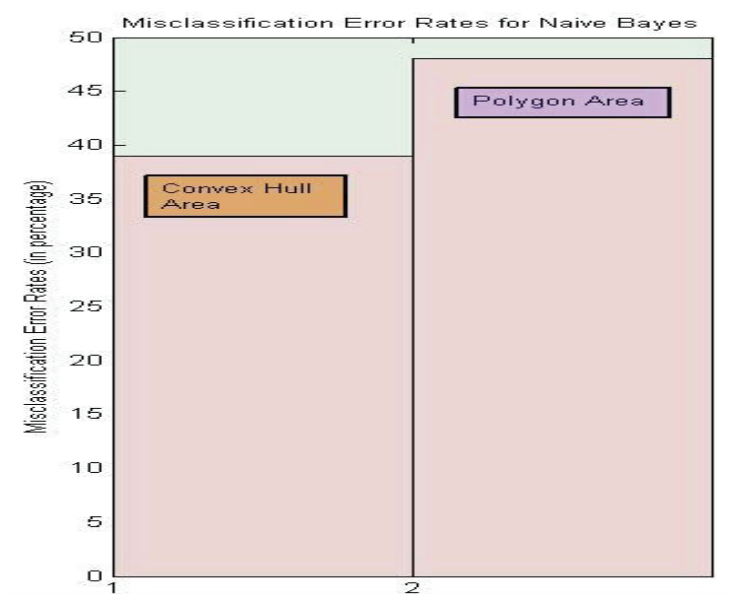

Fig.13. Misclassification error rates for Naïve Bayes' classifier

the distinct clear winner in this case. The mean and standard deviation of misclassification error rates applied for the various distance based classifiers using convex hull area has been shown in Table 13. It has been observed in the cross validation technique that in few cases the classifiers with the highest standard deviation provide a larger variance around the mean. Due to given heterogeneous nature of the data and the inherent bias introduced by the $\mathrm{N}$-fold cross validation technique, we observe that at some speeds the best classifier performs extremely well whereas at other speeds less promising results have been obtained. In 
polynomial based distance metric technique, the misclassification error rates obtained from $\mathrm{n}$-fold cross validation technique has been presented in Fig 15.

In addition to other distance metrics, we have included Minkowski distance with $p=5,6,7$. It has been noted that that all the three Minkowski distances have close misclassification error rates with each other which are higher than the other distance based metrics. The following table 14 indicates statistical measurement of mean and standard deviation for the polynomial based distance metrics.

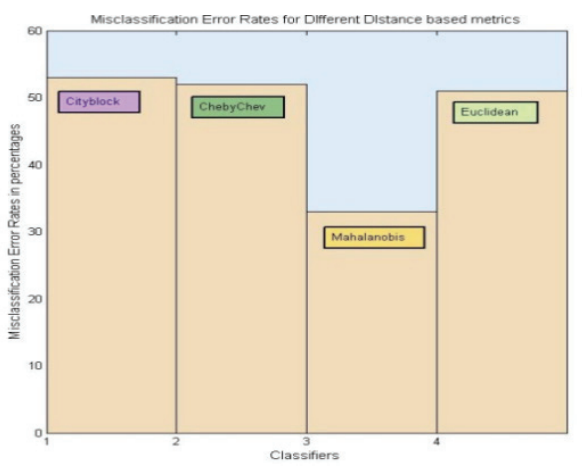

Fig.14. Misclassification error rates of distance based Classifiers

Table 13. Statistical Error measurement for Distance based classifier

\begin{tabular}{lcc}
\hline Distance Metric & Mean & Standard Deviation \\
\hline Cityblock & 53.596 & 5.26 \\
ChebyChev & 52.713 & 7.32 \\
Mahalanobis & 33.67 & 12.85 \\
Euclidean & 53.921 & 6.87 \\
\hline
\end{tabular}

Table 14. Statistical Error measurement for Polynomial based distance metrics

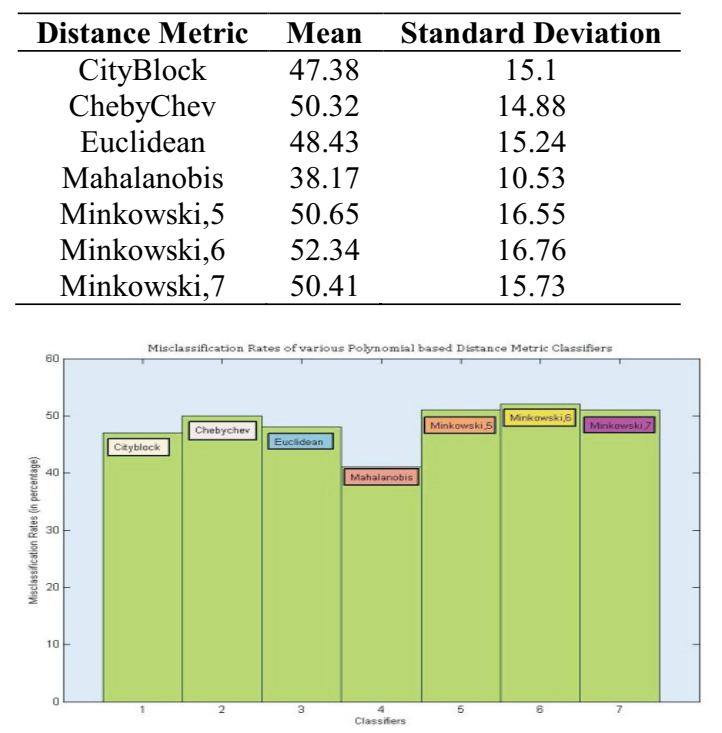

Fig. 15.Misclassification Error rates for polynomial based distance metrics
Table 15. Different Error measurements on Naïve Bayes' rule

\begin{tabular}{ccccccc}
\hline & \multicolumn{3}{c}{ Convex hull area } & \multicolumn{3}{c}{ Polygon Area } \\
\hline $\begin{array}{c}\text { Classifier } \\
\text { Name }\end{array}$ & SSE & MSE & SEM & SSE & MSE & SEM \\
\hline $\begin{array}{c}\text { Naïve } \\
\text { Bayes' } \\
\text { rule }\end{array}$ & 133.3 & 39.21 & 2.26 & $\begin{array}{c}151 . \\
97\end{array}$ & 44.69 & 2.87 \\
\hline
\end{tabular}

Table 16. Different Error measurements on Distance Metrics

\begin{tabular}{|c|c|c|c|c|c|c|}
\hline & \multicolumn{3}{|c|}{ Convex Hull area } & \multicolumn{3}{|c|}{ Polygon Area } \\
\hline $\begin{array}{l}\text { Distance } \\
\text { Metrics }\end{array}$ & SSE & MSE & SEM & SSE & MSE & SEM \\
\hline City Block & $\begin{array}{c}204 . \\
69\end{array}$ & 60.20 & 3.36 & $\begin{array}{c}221 . \\
60\end{array}$ & 65.17 & 3.37 \\
\hline ChebyChev & $\begin{array}{c}196 . \\
44\end{array}$ & 57.77 & 2.40 & $\begin{array}{c}243 . \\
7\end{array}$ & 71.67 & 3.63 \\
\hline Mahalanobis & $\begin{array}{c}141 . \\
72\end{array}$ & 41.68 & 2.30 & $\begin{array}{c}183 . \\
58\end{array}$ & 53.99 & 2.76 \\
\hline Euclidean & $\begin{array}{c}148 . \\
64\end{array}$ & 43.71 & 2.55 & $\begin{array}{c}168 . \\
31\end{array}$ & 49.52 & 2.70 \\
\hline
\end{tabular}

Table 17. Different Error measurements on Polynomial Distance Metrics

\begin{tabular}{ccccccc}
\hline & \multicolumn{3}{c}{ Convex hull area } & \multicolumn{3}{c}{ Polygon Area } \\
\hline $\begin{array}{c}\text { Polynomial } \\
\text { based } \\
\text { metric }\end{array}$ & SSE & MSE & SEM & SSE & MSE & SEM \\
\hline City Block & 252. & 74.4 & 3.41 & 285. & 83.45 & 3.95 \\
& 96 & 0 & & 14 & & \\
ChebyChev & 236. & 69.4 & 3.55 & 261. & 77.05 & 3.57 \\
& 17 & 6 & & 97 & & \\
Mahalanobis & 146. & 43.0 & 2.35 & 169. & 49.70 & 2.64 \\
& 35 & 4 & & 01 & & \\
Euclidean & 180. & 53.1 & 2.87 & 184. & 54.39 & 3.13 \\
& 61 & 2 & & 93 & & \\
\hline
\end{tabular}

The three classification algorithms have been compared and analyzed in the light of different error metrics such as Sum of Squared Error (SSE), Mean Square Error (MSE) and Standard Error of Mean (SEM). The computed Error components for Naïve Bayes' rule, distance based metrics and polynomial based distance metrics have been shown in Table 15, 16 and 17 respectively. It has been observed that less error is incurred for calculation of feature vectors using convex hull methods. The Naïve Bayes' rule and Mahalanobis distance metric produce less MSE and SEM for convex hull method. This comparative analysis will allow us to understand the accuracy level and performance strength of each classifier. 


\subsection{Classifier performance analysis using ROC and CMC analysis}

In order to estimate the sensitivity and specificity of our classifiers, the ROC (Receiver Operating Characteristic) curve has been used to calculate the optimal operating point for each classifier by setting various thresholds. The ROC curve provides an implicit view of the inner workings of the system model. The CMC (Cumulative Match Characteristics Curve) is also illustrated to analyze graphically the performance of all the classifiers.

\subsubsection{ROC Space Analysis}

The Fig.16 shows the ROC space analysis for the Naïve Bayesian classifier. The line $y=x$ often dubbed as the line of no discrimination has been drawn to show the performance of any random classifier which has an equal TPR (True Positive Rate) and FPR (False Positive Rate). Therefore any classifier which has its operating point on the line works no better than a random classifier. A classifier above the line can be dubbed as an optimal classifier and a classifier which is below the line is acting as a sub-optimal classifier. In the Naïve Bayes' example using both the convex hull and the polygon based area, we acquire a classifier which lies above the line of no discrimination. In Fig.16 ROC space for the Naïve Bayes classifier has been illustrated where convex hull area proves to be a better in comparison to the polygon based area. The ROC space for the Distance based classifier and polynomial based classifier has been shown in Fig 17 and Fig.18. It can be inferred from these graphs that the Mahalanobis distance metric is the only optimal classifier and the rest construct a sub-optimal cluster below the line of no discrimination.

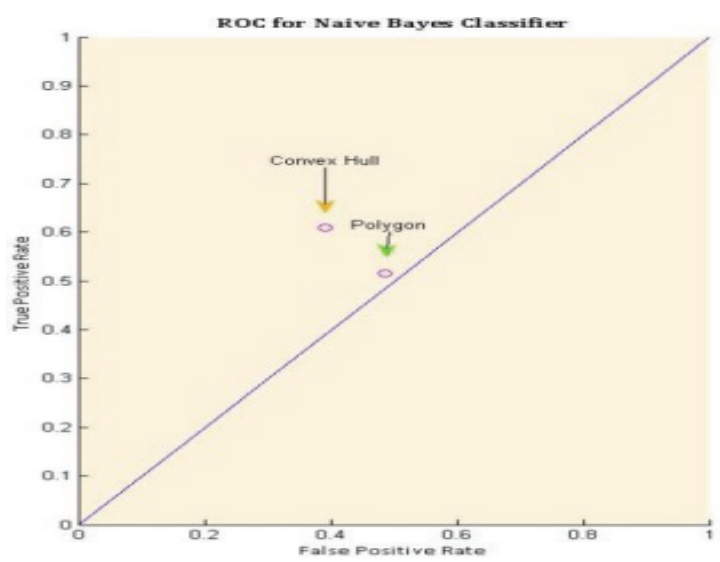

Fig. 16. ROC space for Naïve Bayesian Classifier

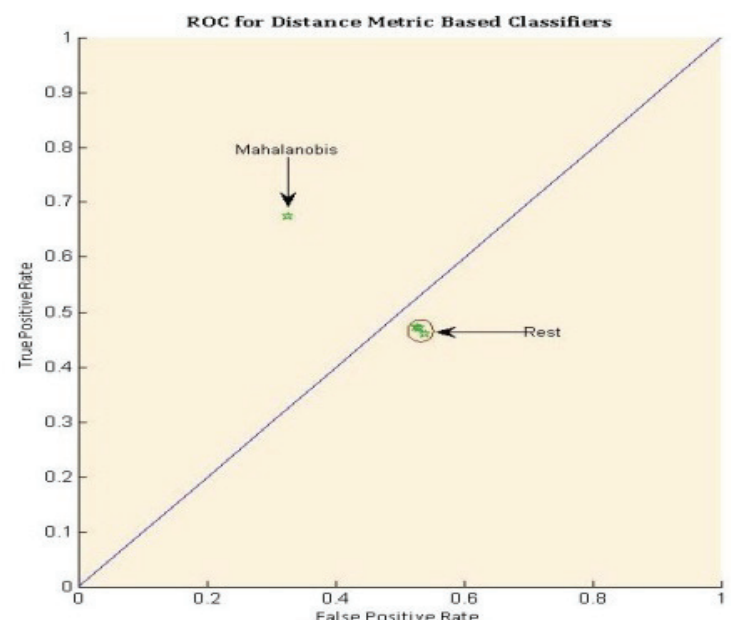

Fig. 17. ROC space for distance based Classifier

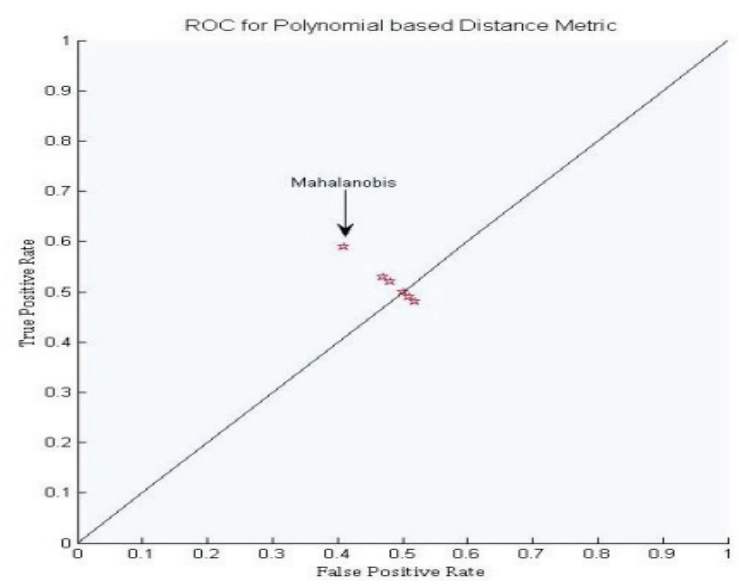

Fig. 18. ROC space for polynomial based distance classifier

\subsubsection{ROC Curve Analysis}

We have plotted the ROC curve for the Naïve Bayes' classifier using different thresholds; the optimal operating point for each classifier has been discussed in earlier figures of the ROC space analysis. Fig. 19 represents the ROC curves for the Bayes' classifier using the polygon and convex hull area. The convex hull area gives better performance in comparison to the polygon area; hence, the Naïve Bayes' classifier for the convex hull area is the clear winner in this regard. 


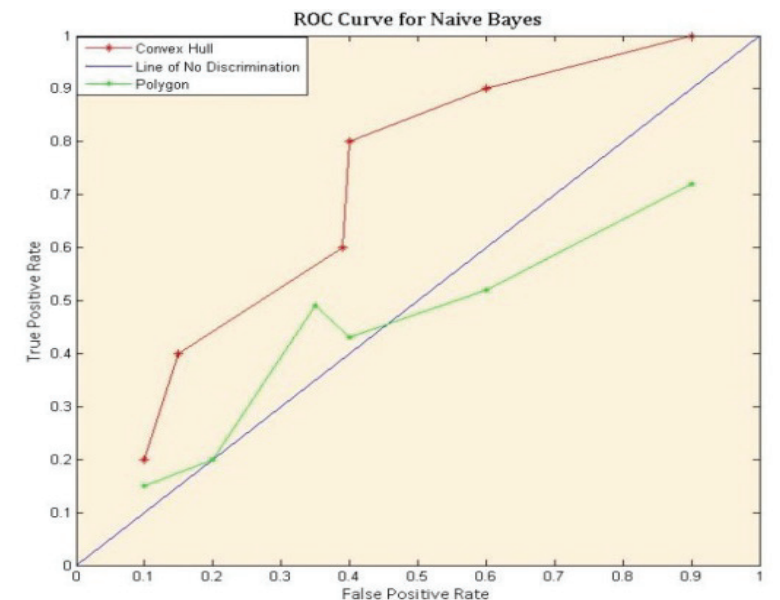

Fig. 19. ROC curve for Naïve Bayes' Classifier

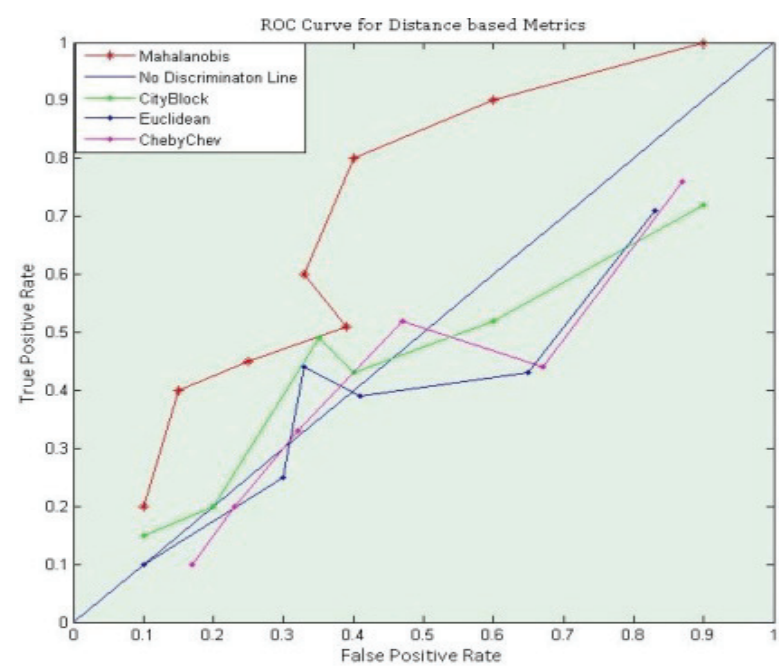

Fig. 20. ROC curve for distance based classifier

The Fig. 20 shows the ROC curve for the distance based metrics. It represents the various thresholds and the corresponding rates of TPR and FPR. The Mahalanobis distance shows the best classification results above the line of no discrimination. Therefore, it is an optimal classifier. It is intriguing to notice that the curve of Mahalanobis is almost similar to Naïve Bayes' using convex hull area. This can be attributed to the fact that we have used different thresholds for them which are introducing a natural bias. The comparison of the three best classifiers has been depicted in Fig. 21 using the three different classification techniques. The convex hull using the Naïve Bayes' rule, the distance metric using Mahalanobis distance, and the polynomial based distance metric using the Mahalanobis distance has been experimentally proved as the best classifiers.

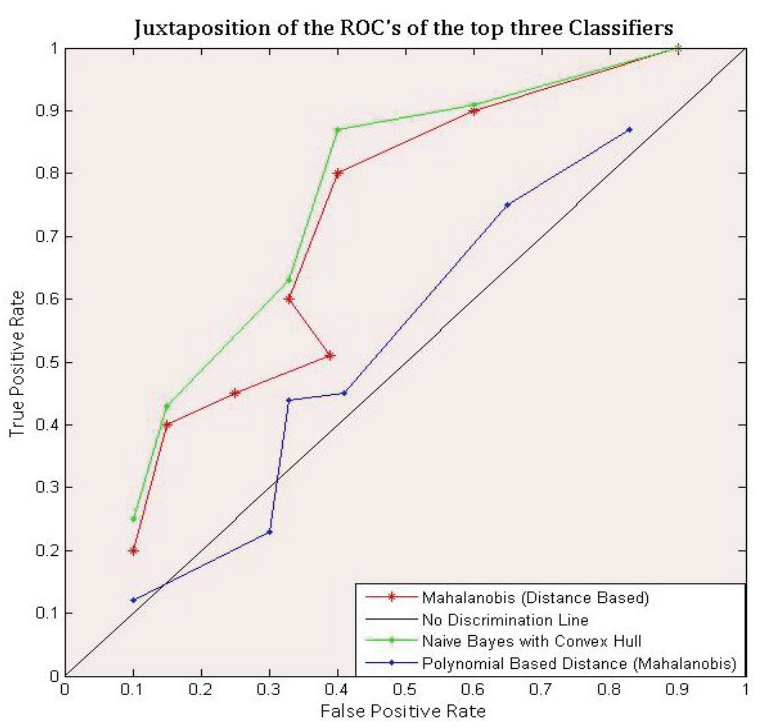

Fig. 21. Comparison of ROC curve for three classifiers

\subsection{CMC results for all the classifiers}

The Fig 22 shows the CMC curve for the different polynomial based classifiers. The CMC for the Naive Bayes' rule has been depicted in Fig 23. The Fig. 24 represents the CMC curves for distance based metrics. It shows the ranks vs. the scores where the scores in this case is the number of times the correct result has come at the top and this is denoted by rank 1 . The number of times the correct result has come within top 2 is included in rank 2 and this process continues to achieve different score results. Therefore, the number of ranks indicates the number of classes (in our case 34) and the score of the last class is always equal to one. The scores have been normalized on a scale of $[0,1]$. In this analysis, the 


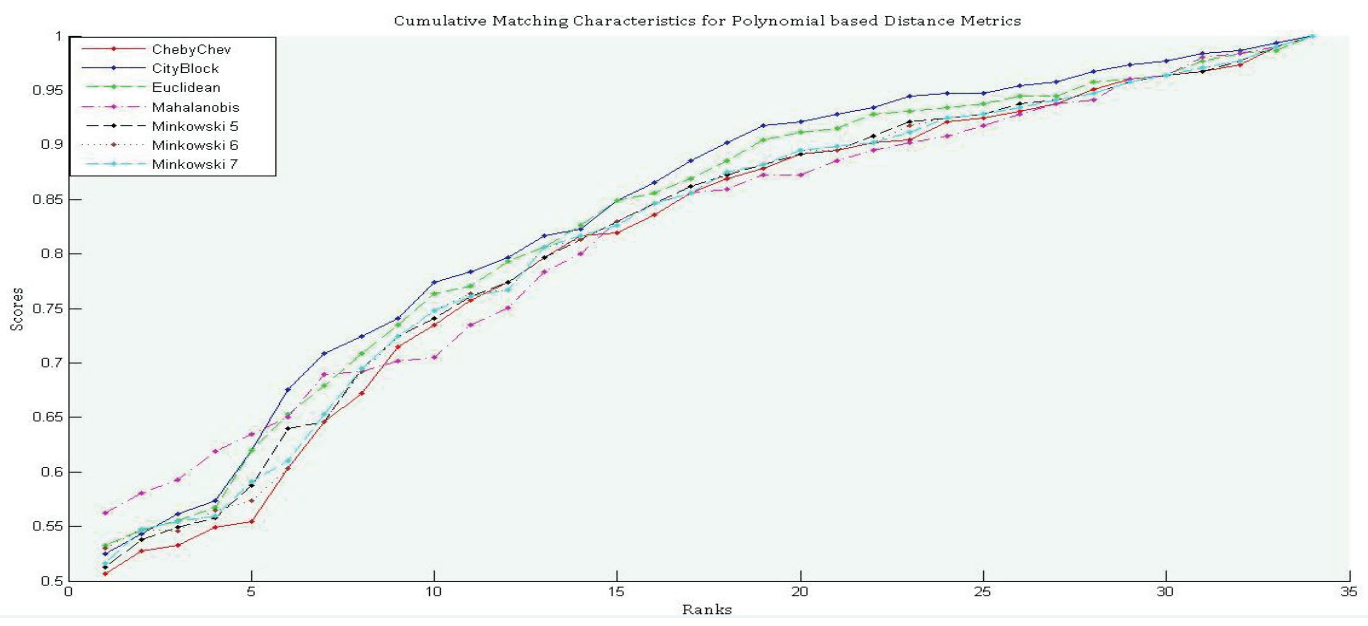

Fig .22. CMC curve for polynomial based Distance based classifiers

convex hull area leads the polygon area so the results we obtained are consistent throughout the process.

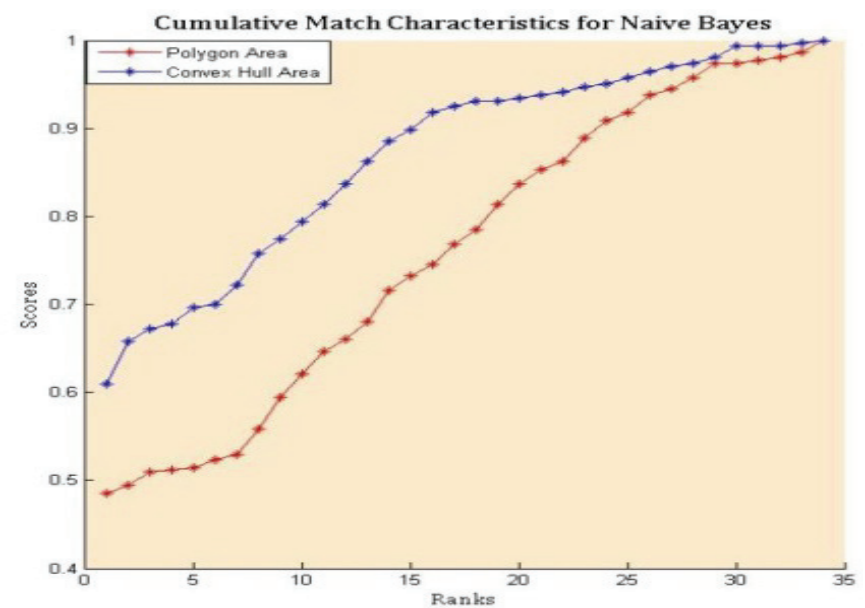

Fig. 23. CMC curve for Naïve Bayesian classifiers

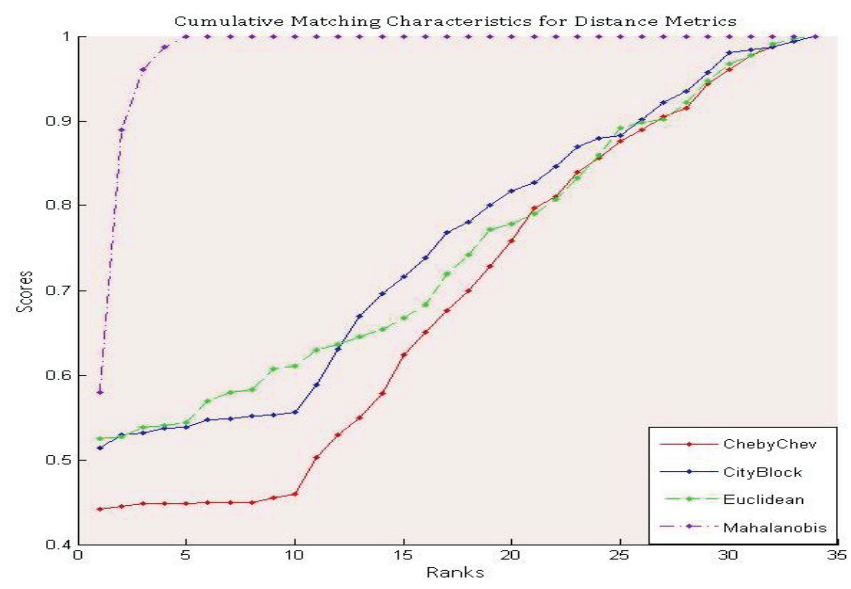

Fig. 24. CMC curve for Distance based classifiers
Since all the three classifiers are base learners or weak learners they can be definitely combined together using ensemble learning paradigms like bagging, boosting and stacking. This should improve the efficacy of the classification results at the cost of increased Computational Complexity, which usually entails such a process. In our case, since all the classifiers are heterogeneous and diverse, they can be ensembled together in a learning model, which has a greater generalization to unseen testing data. However, care must be taken to design the model in such a way that the over fitting is minimal. For this we propose to use stacking with a weighted majority voting to combine the different classification results in order to produce a unique result. Hence, this will result in the creation of a meta-model for the combination of different classification results, which will induce greater diversity in the hypotheses space such that it is able to model the target function better.

In order to perform experimental comparison, no sufficient literature is found which uses our set of features or anything closely related to our approach. The only existing work which is even remotely to our present work has been found in Nixon et. al. [29]. They have proposed a gait recognition technique using area based metrics which uses area based masks to extract the features. Though we are implicitly using the area masks by hierarchically defining the regions of interest for feature extraction, but still it is quite a far cry from their approach. But for reasons of scientific completeness and soundness we have juxtaposed our results in Table 18 using our best results achieved by Bayes' decision rule. 
Table 18. Experimental comparison with Nixon et.al [29 ]

\begin{tabular}{cccc}
\hline & $\begin{array}{c}\text { Recognition rate } \\
(\%) \text { using our } \\
\text { technique }\end{array}$ & $\begin{array}{c}\text { Recognition rate } \\
(\%) \text { using } \\
\text { Nixon's technique }\end{array}$ \\
\hline \multirow{3}{*}{ Full Body } & Convex & Polygon & \\
& Hull & based & 29.2 \\
& 60.32 & 48.56 & \\
\hline
\end{tabular}

\section{Conclusion and Future work}

Human identification using computer vision based technique is indeed a complex problem by nature. Different covariates drastically affect a person's walking pattern which leads to incorrect results in person identification problem. In our research work, we have attempted to provide a feasible solution for human recognition system using possible speed invariant features. The human body has been divided into two parts on the sagittal plane and then feature extraction is carried out in a hierarchical fashion using the area of the polygon and convex hull which is formed by the head, arm and leg motion respectively. We employed a slew of distance metric classifiers together with Bayes' decision rule in order to examine the validity of the projected features. It has been observed that the Mahalanobis distance provides a promising classification result which is rather marginally better than the Naïve Bayes' rule for the calculation of convex hull area. A novel approach for classification using polynomial based distance metric has been proposed to model the system using possible speed invariant features. It has been investigated experimentally that a polynomial equation of 5 degree represents the best fit for each independent feature. Therefore, it is required 204 distinct equations to model the entire training set of 34 subjects with six possible features. Finally, the performance evaluation of all the classifiers has been illustrated using statistical metrics such as Receiver Operating Characteristics (ROC) curve and CMC (Cumulative Matching Curve), the misclassification error rate was calculated after performing $\mathrm{N}$-cross validation technique on all the classifiers.

The drawback of our system model is limited to speed invariant human identification problem which might be infeasible to recognize humans correctly on changing other covariate factors. We have used swing area as a measure of the features which are invariant in the sense that they are carrying unique characteristics for a person at different speeds. We propose to apply Principal Component Analysis (PCA) to reduce the curse of dimensionality problem in high dimensional feature space. This Eigen Space Transformation (EST) will provide greater insights into the interconnections of the features and its possible connotations. In the track of classification methodology, Artificial Neural Network with multilayer perceptron could be applied to solve the uncertainty difficulties in person specific gait signal affected by external and internal factors.

\section{Acknowledgements}

We owe our sincere thanks and gratitude to Prof Yasushi Yagi and his whole research team for sharing with us the valuable OU-ISIR [30] Gait database without which the work could not have been done.

\section{References}

1. F. Jean, A.B. Albu, R. Bergevin, Towards view-invariant gait modeling: Computing view-normalized body part trajectories, Pattern Recognition, 42(11) (2009), pp. 29362949.

2. Z. Xue, D. Ming, W. Song, B. Wan, S. Jin, Infrared gait recognition based on wavelet transform and support vector machine, Pattern Recognition, 43(8) (2010), pp.29042910.

3. K. Bashir, T. Xiang, S. Gong, Gait recognition without subject cooperation, Pattern Recognition Letters, 31(13) (2010), pp. 2052-2060.

4. D.A. Winter. The Biomechanics and Motor Control of Human Movement. John Wiley and Sons, 2nd edition, (1990).

5. University of Glasgow. Definition and description of the gait cycle

www.gla.ac.uk/ibls/us/fab/tutorial/anatomy/hfgait.html, November (2012).

6. M. Murray, A. Drought, R. Kory, Walking patterns of normal men, The journal of bone and joint surgery, 46(2) (1964), pp. 335 -360

7. G. Johansson. Visual perception of biological motion and a model for its analysis. Attention, Perception, and Psychophysics, 14 (1973), pp. 201-211.

8. S. Mondal, A. Nandy, P. Chakraborty and G.C. Nandi. 2012. Gait Based Personal Identification System Using Rotation Sensor. Journal of Emerging Trends in Computing and Information Sciences, 3(2) (2012), pp.395402.

9. L. Lee and W. E. L. Grimson. Gait analysis for recognition and classification. In Proc. Fifth IEEE International Conference on Automatic Face and Gesture Recognition, (2002), pp. 148-155.

10. L. Wang, H. Ning, T. Tan, W. Hu, Fusion of static and dynamic body biometrics for gait recognition, In IEEE 
Transactions on Circuits and Systems for Video Technology, 14(2) (2004), pp. 149-158.

11. C. Ben Abdelkader, R. Cutler, and L. Davis. Stride and cadence as a biometric in automatic person identification and verification. In Proceedings of the Fifth IEEE International Conference on Automatic Face and Gesture Recognition, (2002), pp.372-377.

12. A. F. Bobick and A. Y. Johnson. Gait recognition using static, activity-specific parameters. In Proc. IEEE International Conference on Computer Vision and Pattern Recognition, 1 (2001), pp. 423-430.

13. J. W. Davis and S. R. Taylor. Analysis and recognition of walking movements. In Proceedings. 16th International Conference on Pattern Recognition, 1 (2002), pp. 315-318.

14. C. Yam, M. Nixon, J. Carter, Automated person recognition by walking and running via model-based approaches, Pattern Recognition, 37(5) (2004), pp. 10571072.

15. S.A. Niyogi and E.H. Adelson. Analyzing and recognizing walking figures in XYT. (1994) pp. 469474.

16. D. Cunado, M. S. Nixon, and J. N. Carter. Using gait as a biometric, via phase weighted magnitude spectra. In Proceedings of 1st Int. Conf. on Audio- and Video-Based Biometric Person Authentication, (Springer Verlag, 1997). pp. 95-102.

17. Z. Liu, S. Sarkar, Simplest representation yet for gait recognition: Averaged silhouette, In Proc. 17th Int. Conf. on Pattern Recognition, (2004), pp. 211-214.

18. A. Sundaresan, A. RoyChowdhury, R. Chellappa, A hidden markov model based framework for recognition of humans from gait sequences, In Proc. IEEE Int. Conf. Image Processing (2003), pp. 93-96.

19. W. Liang, T. Tieniu, N. Huazhong, H. Weiming, Silhouette analysis-based gait recognition for human identification, In IEEE Transactions on Pattern Analysis and Machine Intelligence, 25(12) (2003), pp. 1505-1518.

20. J. Little, J. Boyd, Recognizing people by their gait: the shape of motion, Videre:Journal of Computer Vision Research, 1(2) (1998), pp. 1-32.

21. S. Sarkar, P.J., Phillips, Z. Liu, I.R. Vega, P. Grother, K.W. Bowyer, The human ID gait challenge problem: data sets, performance, and analysis, Pattern Analysis and Machine Intelligence, IEEE Transactions on, 27(2) (2005), pp.162-177.

22. Hayfron-Acquah, M. Nixon, J. Carter, Automatic gait recognition by symmetry analysis, Pattern Recognition Letters, 24(13) (2003), pp. 2175-2183.

23. A. Tsuji, Y. Makihara, Y. Yagi, Silhouette transformation based on walking speed for gait identification, In IEEE Conference on Computer Vision and Pattern Recognition (CVPR) 717 (722) (2010), pp. 13-18.

24. W. Kusakunniran, Q. Wu, J. Zhang, and H. Li. Gait recognition across various walking speeds using higher order shape configuration based on a differential composition model. In IEEE Transactions on Systems,
Man, and Cybernetics, Part B: Cybernetics, 42(6) (2012), pp. 1654-1668.

25. Yu Guan; Chang-Tsun $\mathrm{Li}, \mathrm{A}$ robust speed-invariant gait recognition system for walker and runner identification, In International Conference on Biometrics (ICB), 1(8) (2013),pp. 4-7.

26. Y. Makihara, H. Mannami, A. Tsuji, M.A. Hossain, K. Sugiura, A. Mori, and Y. Yagi, The OU-ISIR Gait Database Comprising the Treadmill Dataset," IPSJ Trans. on Computer Vision and Applications, 4 (2012), pp. 53-62.

27. Quick hull algorithm http://www.cse.yorku.ca/ aaw/Hang/quick_hull/Alg orithm.html

28. t-Distribution Table $\mathrm{http}: / /$ onlinestatbook.com/2/estimation/t_distribution.html

29. P. Jeff Foster, Mark S. Nixon, and Adam. Prugel-Bennett, Automatic gait recognition using area-based metrics, In Pattern Recognition Letters, 24 (2003), pp.2489-2497.

30. OU-ISIR Gait Database http://www.am.sanken.osaka-u.ac. jp/GaitDB/index.html 